\title{
New results on equatorial thermospheric winds and the midnight temperature maximum
}

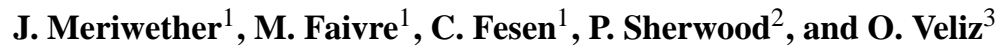 \\ ${ }^{1}$ Department of Physics and Astronomy, Clemson University, Clemson, SC, USA \\ ${ }^{2}$ Interactive Technology, Waban, MA, USA \\ ${ }^{3}$ Radio Observatorio de Jicamarca, Instituto Geofisica del Peru, Lima, Peru
}

Received: 7 August 2007 - Revised: 22 December 2007 - Accepted: 9 January 2008 - Published: 26 March 2008

\begin{abstract}
Optical observations of thermospheric winds and temperatures determined with high resolution measurements of Doppler shifts and Doppler widths of the OI 630-nm equatorial nightglow emission have been made with improved accuracy at Arequipa, Peru $\left(16.4^{\circ} \mathrm{S}, 71.4^{\circ} \mathrm{W}\right)$ with an imaging Fabry-Perot interferometer. An observing procedure previously used at Arecibo Observatory was applied to achieve increased spatial and temporal sampling of the thermospheric wind and temperature with the selection of eight azimuthal directions, equally spaced from 0 to $360^{\circ}$, at a zenith angle of $60^{\circ}$. By assuming the equivalence of longitude and local time, the data obtained using this technique is analyzed to determine the mean neutral wind speeds and mean horizontal gradients of the wind field in the zonal and meridional directions. The new temperature measurements obtained with the improved instrumental accuracy clearly show the midnight temperature maximum (MTM) peak with amplitudes of 25 to $200 \mathrm{~K}$ in all directions observed for most nights. The horizontal wind field maps calculated from the mean winds and gradients show the MTM peak is always preceded by an equatorward wind surge lasting $1-2 \mathrm{~h}$. The results also show for winter events a meridional wind abatement seen after the MTM peak. On one occasion, near the September equinox, a reversal was observed during the poleward transit of the MTM over Arequipa. Analysis inferring vertical winds from the observed convergence yielded inconsistent results, calling into question the validity of this calculation for the MTM structure at equatorial latitudes during solar minimum. Comparison of the observations with the predictions of the NCAR general circulation model indicates that the model fails to reproduce the observed amplitude by a factor of 5 or more. This is attributed in part to the lack of adequate spatial resolution in the model as the MTM phenomenon takes place within a scale of $300-500 \mathrm{~km}$ and $\sim 45 \mathrm{~min}$ in local time.
\end{abstract}

Correspondence to: J. Meriwether

(john.meriwether@ces.clemson.edu)
The model shortcoming is also attributed in part to the need for the model to include a hydrodynamical mechanism to describe the merging of the zonal wind with the meridional tidal winds that converge onto the geographical equator. Finally, a conclusion of this work is that the MTM compressional heating takes place along the perimeter of the pressure bulge rather than within the bulge, an issue previously not appreciated.

Keywords. Atmospheric composition and structure (Airglow and aurora) - Meteorology and atmospheric dynamics (Thermospheric dynamics; Instruments and techniques)

\section{Introduction}

The midnight temperature maximum (MTM) is a low latitude thermospheric phenomenon that has a long history in aeronomy from the first detection in the early 1970s. Modeling the MTM nighttime behavior and its structure still remains a challenge with little success achieved thus far. The new results reported in this paper show how the equatorial thermosphere dynamics contribute to the development of the MTM phenomenon. None of the current physics-based numerical models is able to reproduce the MTM phenomenon with the amplitudes of 150-200 K that are commonly observed with the Arequipa Fabry-Perot interferometer (Faivre et al., 2006). The implication of the inability of grid point models such as that of the NCAR TIME-GCM (Roble et al., 1988, 1994, 1996) to reproduce the MTM appearance indicates that the ion-neutral coupling between the low-latitude F-region plasma and the thermosphere is poorly understood, and the crucial interplay of the neutral dynamics with the background ionosphere is not being represented correctly within the model. The MTM represents an excellent test of current physics-based models and how well they simulate the low latitude thermosphere and ionosphere. The lack

Published by Copernicus Publications on behalf of the European Geosciences Union. 
of success in modeling the MTM clearly has implications relating to the application of such models for space weather forecasting.

In this paper we present new Arequipa results on the latitudinal distribution of the thermospheric winds observed at times when MTM events have been detected. Data acquired with the high-resolution Fabry-Perot interferometer (FPI) operating at Arequipa, Peru $\left(16.2^{\circ} \mathrm{S}, 71.5^{\circ} \mathrm{W}\right)$ are analyzed to yield 630-nm airglow intensity, thermospheric line-of-sight wind and temperature. Each of these three quantities is a column average over the line-of-sight path through the $630-\mathrm{nm}$ nightglow layer generated by the F-region nighttime photochemistry. The peak emission altitude of the $630-\mathrm{nm}$ volume emission profile varies between $240 \mathrm{~km}$ (solar minimum) and $265 \mathrm{~km}$ (solar maximum), depending upon solar flux activity.

The increase in sensitivity achieved by the replacement of the photomultiplier detector with a cooled bare CCD camera exceeded one order of magnitude, thus significantly improving the temporal resolution and measurement precision for Arequipa FPI observations beginning in 2005. Imaging Fabry-Perot interferometer observatories equipped with good quality CCD detectors operating in the polar region have also experienced a similar rise in sensitivity. The new results achieved combined with earlier work using the technology of image plane detectors have produced significant advances in the understanding of the polar thermospheric dynamics (Aruliah and Griffin, 2001; Aruliah et al., 2005). One remarkable highlight of the European recent work using the bare CCD detector is the detection of transient responses of the thermosphere caused by abrupt changes in the ion-neutral coupling.

\section{Summary of the MTM phenomenon}

The MTM peak and its linkage to a simultaneous thermospheric nighttime meridional wind reversal were first detected with ground-based optical and radar instruments (Harper, 1973; Behnke and Harper, 1973; Burnside et al., 1981; also see review by Herrero et al., 1993). Examination of the low-latitude ionosonde data by Nelson and Cogger (1971) revealed that the height of the F-layer often displayed a sudden descent of 50 to $100 \mathrm{~km}$ during the period 00:00 to 02:00 LT. This descent was found to be coincident with a reversal of the meridional wind, as inferred from radar measurements of ion velocities by Harper (1973). The phenomenon was also observed in situ with the Neutral Atmospheric Temperature Experiment flown on the Atmospheric Explorer E satellite (Spencer et al., 1979). The global and seasonal phenomenology have been described by Herrero and Spencer (1982), and Herrero et al. (1983, 1993). Faivre et al. (2006) studied the MTM climatology for the years covering the transition from the solar minimum in 1996 to solar maximum, 2001. This work demonstrated that there was little dependence of the MTM amplitude on the phase of the solar cycle, and found a seasonal variation consistent with
Herrero et al. (1993) with the MTM appearance occurring later at night for the winter than for the equinoxes.

The convergence of thermospheric tidal winds at the equator near local midnight causes a density maximum, and consequently the MTM, through adiabatic heating (Herrero, 1983; Colerico et al., 1996, 2002, 2006; Fesen et al.,1996; Arduini et al., 1997; Faivre et al., 2006). The observed increase can be as much as $150-200 \mathrm{~K}$. A so-called "pressure bulge" generated at the location of the MTM formation near the geographic equator would cause an abatement or reversal of the meridional wind through back pressure.

The modification of the meridional wind component induced by the passage of the pressure bulge transports the plasma within the MTM region to lower altitudes creating enhanced nightglow through the increased recombination of the plasma. This enhanced 630 -nm signature, called a "brightness wave" (BW) by Colerico et al. (1996), has been seen with all-sky imaging observations for stations located as far south as El Leoncito, Argentina $\left(31.8^{\circ} \mathrm{S}, 69.0^{\circ} \mathrm{W}\right)$ and as far north as Arecibo, PR $\left(18.3^{\circ} \mathrm{N}, 66.76^{\circ} \mathrm{W}\right)$ (Martinis et al., 2006; Colerico et al., 2006). The MTM phenomenon is usually observed near midnight or the early morning hours. When the MTM is detected visually through the feature of the $\mathrm{BW}$, it is seen to propagate from west to east and from the equator toward the northern or southern polar region.

Results from NCAR TIEGCM modeling (Fesen, 1996; Colerico et al., 2003, 2006) generally fall short by about a factor of 5 in reproducing the 150 to $200 \mathrm{~K}$ MTM amplitude reported by Faivre et al. (2006). Colerico et al. (2006) noted that what appears to be missing in the modeling work is an adequate prescription of the tidal forcing, namely, the lack of the terdiurnal tidal wave at the model lower boundary.

\section{New observing approach to MTM studies}

Improving our understanding of the relationship between the tidal forcing and the resultant formation of the MTM requires experimental studies designed to observe the MTM spatial structure and its relationship to the tidal dynamics. However, such measurements must have improved temporal sampling of the complex wind and temperature fields, combined with increased spatial resolution, to be useful for comparison with the modeling results. The improved Arequipa FPI sensitivity achieved by replacing the photomultiplier with the CCD camera has made this possible, even with the much weaker solar minimum 630-nm intensities.

Accordingly, we adopted a new observing strategy for the Arequipa FPI similar to that used by Burnside et al. (1981), who studied the MTM dynamics at Arecibo Observatory with observations in 1979 and 1980. Their approach featured an increase in the number of azimuthal directions from the 4 cardinal directions to 8 directions chosen at $45^{\circ}$ intervals from $0^{\circ}$ to $360^{\circ}$. The Burnside et al. results were obtained during solar maximum when the $630-\mathrm{nm}$ intensities 


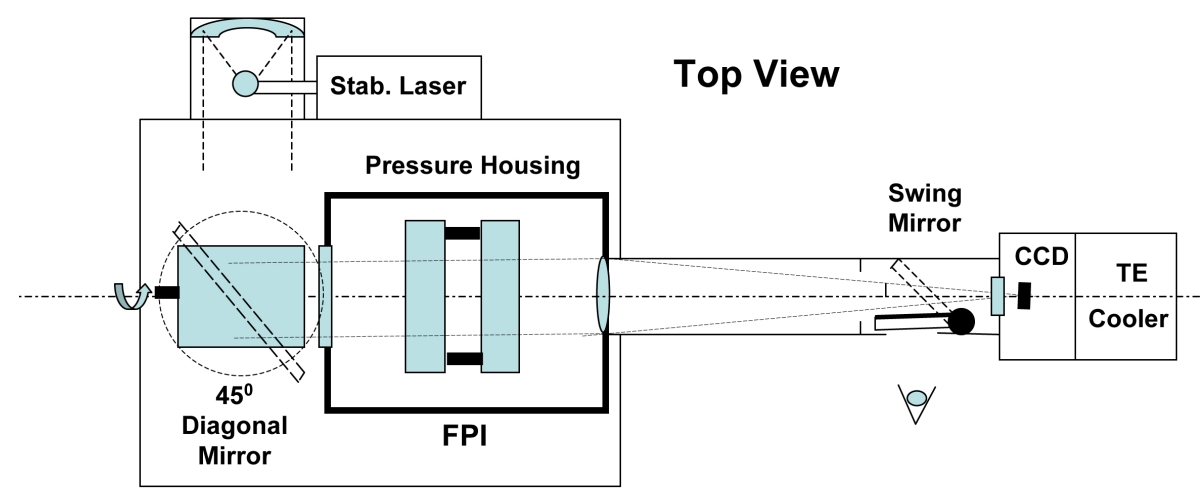

Fig. 1. Schematic of the Arequipa Fabry-Perot interferometer instrument after replacement of the photomuliplier with a bare CCD detector. The diagonal mirror shown is rotated $90^{\circ}$ about the horizontal axis to shift the CCD camera view from the sky to the calibration chamber or the reverse as selected by the computer. The swing mirror allows a view of the etalon chamber while adjusting the etalon plate alignment.

were at least five times brighter than those observed at Arequipa during solar minimum. Moreover, the Burnside et al. wind measurement precision for each direction was more typically $\sim 25 \mathrm{~ms}^{-1}$ for $120 \mathrm{~s}$ integration, in contrast to the $\sim 7-10 \mathrm{~ms}^{-1}$ precision found for the improved Arequipa FPI using the same exposure time, $120 \mathrm{~s}$, during the 2005-2006 nearly solar-minimum observations.

In the analysis of the data acquired by this technique, neutral wind maps are generated by assuming that the temporal variation of the neutral wind field in the zonal direction depends only on the local time over an interval of $30 \mathrm{~min}$. These maps portray the divergence and vorticity of the thermospheric wind field over a latitudinal extent of $8^{\circ}$. Once the divergence has been determined, the vertical wind may be estimated. An interesting finding reported by Burnside et al. (1981) was the correlation of downward vertical winds of 2-5 ms ${ }^{-1}$ with the period of MTM heating, but this has not been confirmed in our analysis of the Arequipa data.

In summary, the new observing technique improves the ability to track the propagation of the MTM and to measure its impact on the neutral thermosphere by using maps of the thermospheric winds observed. These maps can be compared with the onset of the MTM peak structure and with the temporal variation of the inferred vertical velocities calculated from the divergence. In this paper we describe the first results obtained with the modified Arequipa FPI in 2005 and 2006 utilizing this approach.

\section{Instrumental details}

The Arequipa FPI described by Biondi et al. (1985) and Meriwether et al. (1986, 1997) was modified in February 2004 by the replacement of the GaAs photomultiplier detector with a bare CCD camera (Andor Model DU412, with a square chip of $24 \mu \mathrm{m}$ pixels in a $512 \times 512$ array). The images were acquired with $2 \times 2$ binning to improve the signalto-noise ratio. A schematic diagram of the modified instrument is given in Fig. 1. The FPI etalon is housed in a temperature-controlled, sealed chamber filled with argon gas. It has a clear aperture of $10 \mathrm{~cm}$, a spacing of $1.0 \mathrm{~cm}$, and an objective lens focal length of $54.5 \mathrm{~cm}$. The etalon fringe pattern is focused on the CCD chip, which is cooled by a fourstage thermoelectric cooler to $-85^{\circ} \mathrm{C}$ to decrease the dark signals to less than 0.0005 counts per pixel per second, considerably less than the unwanted background from the neighborhood lights. An automated filter changer (not shown) was added to the optical path between the pointing head and the $45^{\circ}$ mirror (shaded rectangle), so that other spectral emissions besides atomic oxygen 630-nm can be observed.

For nightglow observations, the line-of-sight of the instrument is directed upward by the diagonal mirror through a vertical tube (light dashed circle) to the two-axis (Az/El) pointing head on the observatory roof. For instrument finesse determinations, the diagonal mirror is rotated $90^{\circ}$ (dashed rectangle) to view the expanded beam from a stabilized $\mathrm{HeNe}$ laser. Visual alignment of the etalon plates is achieved by rotating the silvered-glass swing mirror $45^{\circ}$ to view the laser fringe pattern. The pressure of the argon gas in the pressure housing is adjusted for optimum placement of the interference fringes on the CCD.

The datataking software used previously (Meriwether et al., 1997; Faivre et al., 2006) was completely rewritten, with four intercommunicating processes providing enhanced capabilities. The MASTER process interprets a user-written script language to provide instructions for the other three processes. The script language is similar to a subset of $\mathrm{C}$ with added statements to operate the equipment. The OBSERVATORY process executes instructions from MASTER to operate the pointing head, and similarly, the CAMERA process 

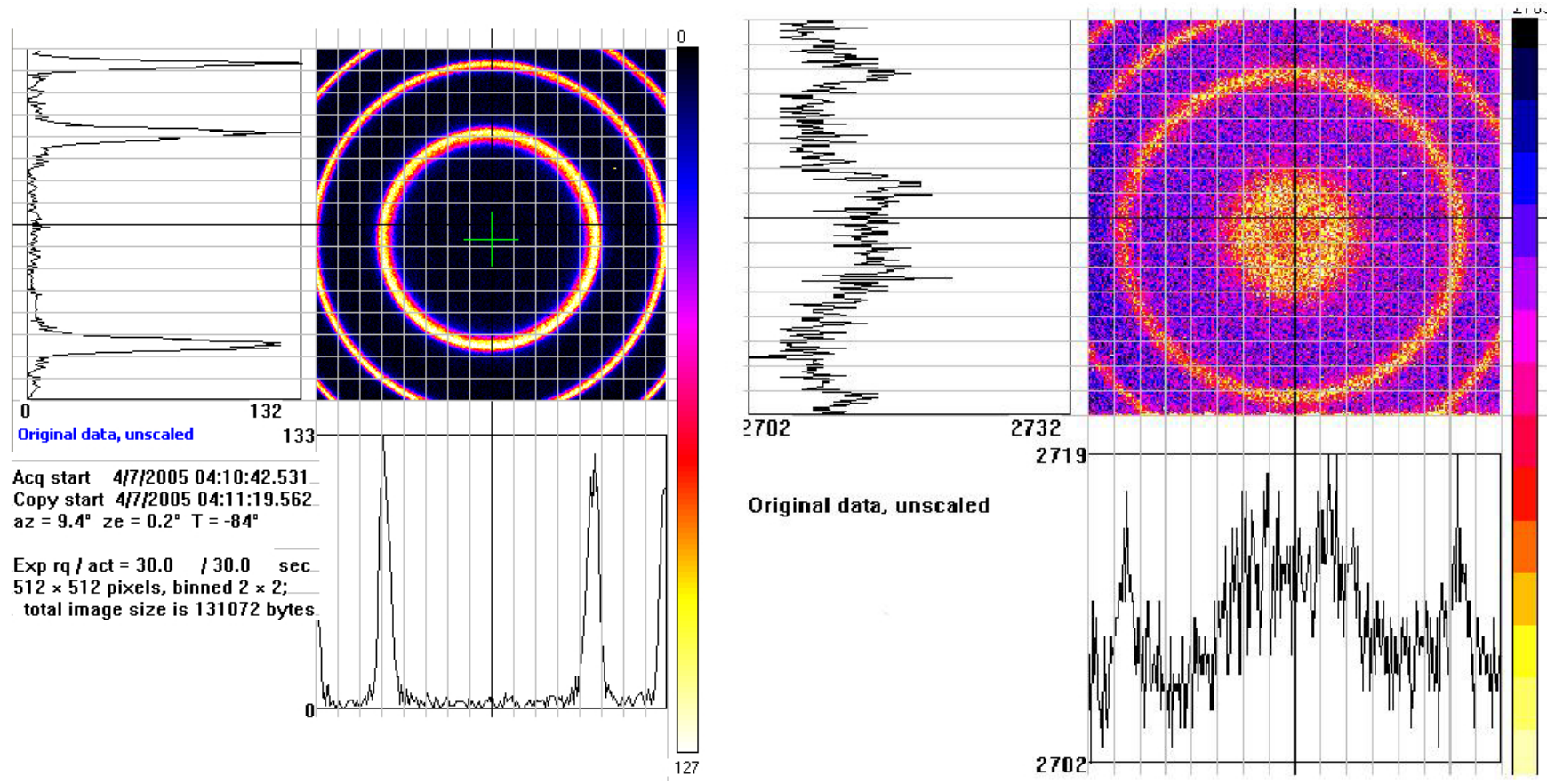

Fig. 2. Image comparison of the HeNe laser (left) and 630-nm nightglow (right) interference patterns plotted by Analyze for observations obtained on 7 April 2005. Vertical and horizontal cross-sectional cuts of the images are plotted to the left of and below each image. The green cross in the laser image represents the chip center. A dark count image has been subtracted from the laser image.

operates the Andor CCD camera. Finally, ANALYZE plots the image data and provides a quick-look view of Doppler shifts and 630-nm intensities. Ephemeris calculations using the Naval Observatory code are performed by MASTER to determine the times for datataking start and stop for each night. MASTER can also calculate the azimuth and zenith angle of the Sun, Moon, and any of the planets at any time to help calibrate the pointing of the pointing head. At the close of each night of observations the images are compressed and transferred by ftp to the analysis computer.

Figure 2 shows examples of images obtained from a $\mathrm{HeNe}$ frequency-stabilized laser and from the 630-nm nightglow. For a nightglow image with an exposure time of $120 \mathrm{~s}$, which was used for the results described below, the difference between peak and background for the $2 \times 2$ superpixels is typically 10 to 12 counts. Each observing cycle included a dark image, a laser calibration image, and images for 2 zenith (vertical) and 8 azimuthal directions from $0^{\circ}$ to $360^{\circ}$ at $45^{\circ}$ intervals. The zenith angle used was $60^{\circ}$. No observations were taken within a $45^{\circ}$ cone centered on the Moon. This precaution was adopted to avoid possible damage to the CCD detector; thus, operations of the Arequipa imaging FPI can be continued through the full moon period. Scattered light from the Moon does not present a problem, although the background continuum increases.

In summary, the modified FPI applied to the observation of a $\sim 50$ Rayleigh $630-\mathrm{nm}$ nightglow signal using $2 \times 2$ binning to produce an image with $256 \times 256$ super pixels indicate that the wind speed and the temperature can now be determined with uncertainties of 7-8 $\mathrm{ms}^{-1}$ and $20-30 \mathrm{~K}$, respectively, in a 120 -s exposure.

\section{Data analysis: determination of the spectrum}

The Arequipa nightglow images are analyzed using the method of annular summing spectroscopy described by Coakley et al. (1996), Mierkiewicz et al. (2006), and Nossal et al. (2006). By imaging the FPI circular interference pattern onto the face of the thermo-electrically cooled bare CCD chip (high quantum efficiency) and then integrating the nightglow images around the center of this pattern to convert the image into a spectrum, the ratio of signal to noise is much improved. The application of annular summing spectroscopy to analyze 630-nm nightglow images utilizes the fact that, for the FPI, equal-area annuli correspond to equal-interval spectral elements when measured in wavelength. However, as noted by Conde (2002), optical aberration would affect the circularity of each order. Each of the two orders in the HeNe laser image was examined at different phases of the interference pattern (by changing the etalon pressure) to detect deviations from circularity. No significant distortion was found, and therefore the analysis procedure becomes greatly simplified. This illustrates the advantage of using a high $\mathrm{f}$-number 
(the ratio of focal length to aperture) for the basic optical design. The optical aberration for a good quality lens becomes less significant as the f-number is increased. The f-number for the Arequipa FPI is 6.3.

The laser calibrations and 630-nm nightglow images were first processed to remove "hot" pixels (pixels with high counts caused by cosmic rays or chip defects). These were identified by searching for those pixels for which the observed response (counts) was $\geq 3$ standard deviations above the average of the eight neighboring pixels. The high value was then replaced by the average. There might be as many as 15 such pixels in any one image.

This step was followed by the removal of the dark signal and bias background. First, all of the dark count images obtained were averaged. Then, to avoid possible effects caused by slow temporal changes in the dark count contribution or the bias, the averaged dark image was scaled to the averaged value of the central pixel region $(100 \times 100)$ in each cycle's dark image. This corrected averaged dark image was subtracted from each of the nightglow images in a given observing cycle (10 directions). This procedure reduces the noise that is introduced by the subtraction of each dark image from the 630 -nm nightglow images.

For the annular summing conversion of the detected image into a spectrum, the center of the circular interference pattern must be accurately determined, and laser images were used for this purpose. The center of each laser image was determined by using cross-sectional cuts at $15^{\circ}$ azimuthal intervals through the image center to get 24 spectra with peaks for each order, as shown for the two cross-section cuts plotted in Fig. 2. The data for each spectrum were searched within the innermost order to find the two peaks, one on each half of the slice relative to the center. An estimate of each peak position along the slice direction was determined by a Gaussian fit to the peak. Because the image is circularly symmetric, each pair of corresponding peak positions determines the pattern center. The 24 sets of center coordinates determined by this procedure were averaged to obtain the final estimate of the pattern center coordinates. The results over successive laser images showed that the precision of the center position determined was $\leq 0.1$ super pixel. The long-term drift over a tenhour period caused by room temperature changes was found to be less than 0.3 super pixel.

The next step in the analysis is the annular summing procedure. Following the recommendations of Coakley et al. (1996), the maximum circular area of the nightglow image, $A_{0}$, is divided into $K$ equal-area annuli. The choice for $K$ must satisfy the constraint that a sufficient number of spectral elements be chosen to ensure adequate sampling for each order (Hernandez, 1986), i.e. greater than 14, to avoid aliasing. This choice must also satisfy the constraint that the cross-sectional width of each annular spectral ring is at least 2 pixels (Coakley et al., 1996), again, to ensure adequate sampling to avoid aliasing. Consequently, we chose the number of spectral elements for each order to be $\sim 17$ and $\mathrm{K}$ would be $\sim 35$ since there are $\sim$ two orders imaged onto the CCD chip. For this value, the cross-sectional widths for the outermost annular ring in the first and second orders are 3.2 and 2.3 super pixels, respectively.

By dividing the area of $A_{0}$ by $K$, the area of each annulus, $A_{i}$, is determined. This is then used to determine the number of pixels for each annulus by dividing this value by the area of each superpixel. The free spectral range for one order was determined by fitting Gaussians to each laser peak of the onedimensional interferogram so that the spectral separation in annuli units could be determined from the peak positions of the two Gaussians.

The radius of the $i$-th outer annulus, $r_{i}$, is determined using the formula $r_{i}=\sqrt{i A_{i} / \pi}$. The counts for all pixels within the annular spectral element defined by the $r_{i}$ and $r_{i-1}$ boundaries are added to the counts of those pixels that are intersected by the inner or the outer radial boundary of each spectral element to get the total signal for that spectral element. The number of pixels added for a spectral element using this rule is compared with the number of pixels corresponding to $A_{i}$. The total count generated in this summing operation is corrected by the ratio of $A_{i}$ to the area corresponding to the number of pixels summed. This normalization factor may vary from unity by a few percent.

As a check, we tried an alternative procedure of dividing each superpixel area $(48 \mu \times 48 \mu)$ overlapped by the annular ring boundary corresponding to $r_{i}$ into $8 \times 8$ mini-pixels, each with the number of counts determined by dividing 64 into the superpixel count signal. These mini-pixels are assigned to one side or the other of the ring boundary between two annular elements and included in the summing operation for one or the other of the two spectral elements. This refinement did not alter the previous results in any significant way. The counts for all of the pixels in each of the $\sim 17$ concentric rings used for each order are summed to obtain a spectrum as counts versus spectral channel number. Examples of the laser and nightglow spectra are presented in Fig. 3. Each peak in the nightglow spectrum may be analyzed to determine the Doppler shift and Doppler broadening of the 630-nm line.

In summary, the application of the imaging CCD detector greatly improves the ratio of signal to noise, $\sim 15 \times$, for the spectral profile of the $630-\mathrm{nm}$ emission as compared with the results from the previous mode of pressure scanning using a photomultiplier for the detector (Meriwether et al., 1997; Faivre et al., 2006).

\section{Data analysis: determination of Doppler shifts and Doppler widths}

Because the transmission of the optics decreases in the radial direction relative to the optical axis, a correction to adjust for this reduction of signal, which might be 10 to $15 \%$, is necessary. There is also the possible reduction of the signal caused by the incidence angle not being perpendicular to the surface 

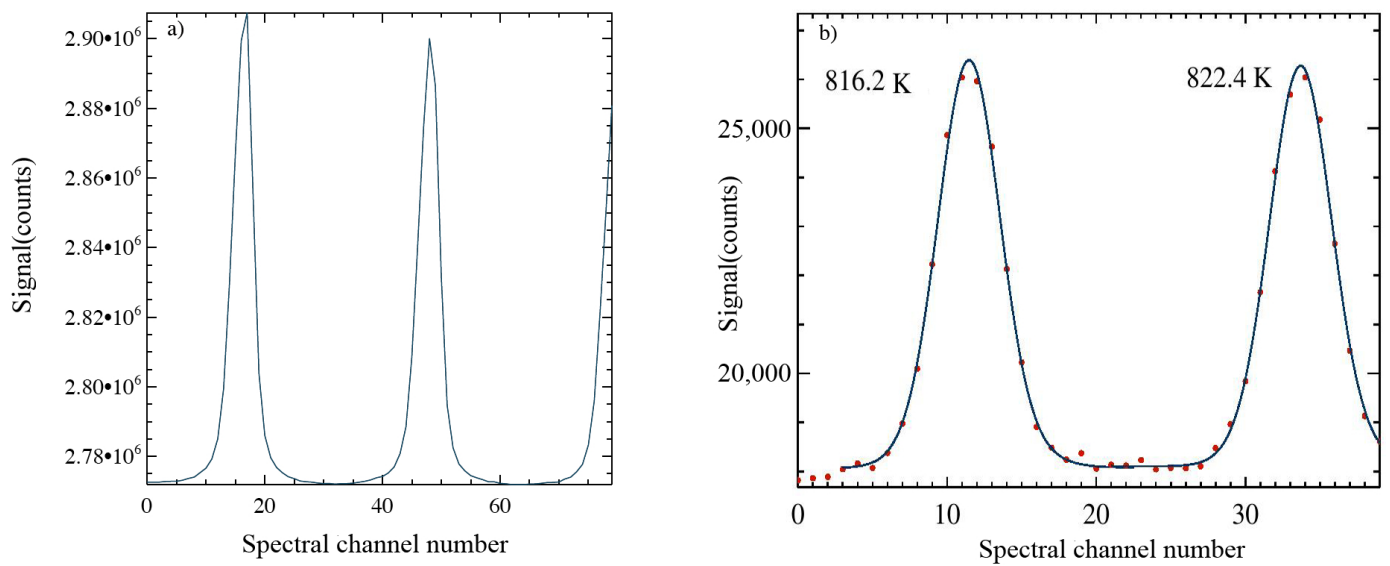

Fig. 3. Two examples of summed spectra for the laser (a) and 630-nm nightglow images (b) obtained for observations on 7 April 2005 . The exposure time for each image was $240 \mathrm{~s}$. The red dots in the nightglow spectrum represent the signal determined by the addition of the pixel signals within each equal-area annulus. Seventeen data points were used in the analysis for each order. Temperature values determined by the fit are indicated in the figure as $816.2 \mathrm{~K}$ and $822.4 \mathrm{~K}$. Ninety annular rings were used for the laser plot to illustrate the line shape more precisely; in the Fourier analysis described in the text, the laser profile would be determined using 17 annular rings. The finesse for the laser interferogram is $\sim 8.0$.

of the interference filter for the outermost annular elements. A correction function can be determined by the method of flat-fielding, as described by Coakley et al. (1996), in which an uniform continuum source, such as early twilight, is observed to measure the variation of the signal across the image. This correction is then applied to the two-dimensional image before the annular summing operation is applied.

An alternative approach that we chose to use is to determine the signal decrease observed in the laser spectrum, for which the intensity decreases linearly from the innermost order to the outermost order. The slope of this decrease is used to correct the spectral shape, using the fact that each laser fringe should have equal intensities, provided that the source, the laser scattering chamber, is uniformly illuminated. For the inner order, the possible errors for the Doppler broadenings and Doppler shifts following this approach are estimated to be less than $10 \mathrm{~K}$ and negligible, respectively; the temperature for the outer order was found to be $\sim 30 \mathrm{~K}$ higher without this correction, but there was no change in the inferred Doppler shift. A justification for using this method rather than the first approach is that the image for early twilight will have Fraunhofer structure that may affect the function determined. There is also the possibility of a need to correct for the non-linearity of the detector if the twilight continuum intensity is too bright. For the approach used to be valid, it is necessary that the position of the interference image be adjusted to have the ring center of the interference pattern approximately coincide with the optical axis. This avoids any problems that might be introduced by the radial symmetry of the optical attenuation.

This step of correcting the spectral shape by this linear function is followed by the analysis of the spectral profile, which is performed by using the technique developed to analyze the Arequipa pressure scanning observations of the past (Meriwether et al., 1986, 1997; Faivre et al., 2006). This analysis procedure is based upon a non-linear leastsquares fitting procedure that is referred to as the LevenbergMarquardt algorithm (Bevington and Robinson, 1992). The $\mathrm{O}\left({ }^{1} \mathrm{D}\right)$ nightglow spectral emission profile is modeled as a Gaussian source with four parameters: the spectral background (starlight and dark count), the 630-nm spectral intensity, the Doppler broadening width, and the 630-nm peak position (Hays and Roble, 1971; Killeen and Hays, 1984).

The Fourier transform representation of the FPI instrument function is determined from the laser image, and this function is applied to each of the two fringes of the nightglow spectrum (Fig. 3b). The sine and cosine coefficients of a complete Fourier series are used to represent the instrument function over one free spectral range, as described by Meriwether et al. (1997). Data analysis values for the four spectral parameters are generated by application of the LevenbergMarquardt fitting algorithm to the observed 630-nm spectral profile. Trial values are varied to minimize the differences between the data and the model. In Fig. 3b, the results of this model fitting are compared with the spectral data points, and there is excellent agreement between the fit derived and the observations.

The line-of-sight wind speeds are then determined from the $630-\mathrm{nm}$ peak positions using the determined zenith 630$\mathrm{nm}$ peak position for each cycle as the zero Doppler shift reference throughout the night. This approach is based upon the fact that generally the amplitude of the vertical wind is small $(\sim 1 \%)$ when compared with typical horizontal speeds. A spline approximation is applied to the set of zenith peak 
positions and times so that the reference zenith position at the time of each azimuthal direction can be estimated with an uncertainty of several $\mathrm{ms}^{-1}$. For each of the eight azimuthal directions, the line-of-sight speed at a given time is equal to the offset between the peak position determined from the analysis and the reference zenith position inferred from the spline fit. These Doppler shift speeds are then increased to estimate the horizontal wind component by the inverse of the sine of the zenith angle. Typical standard deviations found in this analysis for an exposure time of $120 \mathrm{~s}$ and a signal intensity of $\sim 50$ Rayleighs are $\sim 7-10 \mathrm{~ms}^{-1}$ and $20-30 \mathrm{~K}$.

\section{Horizontal wind analysis}

Our analysis to determine the horizontal wind speeds from the line-of-sight (LOS) winds follows the steps described in Burnside et al. (1981), which is based on the decomposition of the line-of-sight winds by a Fourier series truncated at the second order. As indicated in Sect. 1, zenith measurements are made twice in each cycle to derive the zero velocity Doppler reference, and consequently, instrumental drift in the FPI during the night should not affect the Doppler shift determinations. An additional assumption is that the height of the 630-nm nightglow layer does not change over the regions of the sky under observation, which is generally true, except near twilight. Neglecting the effects represented by the curvature of the Earth's surface, the wind vector can be expanded in a Taylor series relative to the zenith of the observation site:

$U_{x}=u_{0}+\frac{\partial u}{\partial x} x+\frac{\partial u}{\partial y} y$
$U_{y}=v_{0}+\frac{\partial v}{\partial x} x+\frac{\partial v}{\partial y} y$

where $u_{0}$ and $v_{0}$ are the zonal and meridional winds in the zenith. This coordinate system uses $x, y$, and $z$ to represent the zonal, meridional, and vertical directions, with positive values to the East, North, and upward, respectively. The LOS speed measured by the instrument is given by

$U_{r}=\sin \theta\left(U_{x} \sin \phi+U_{y} \cos \phi\right)$

with $\theta$ and $\phi$ (measured clockwise from the $y$ axis) the zenith and azimuth angles, respectively. Substitution of Eqs. (1a) and (1b) into Eq. (2) gives the result

$$
\begin{aligned}
\frac{U_{r}}{\sin \theta}= & \frac{R}{2} k_{1}+u_{0} \sin \phi+v_{0} \cos \phi \\
& +\frac{R}{2}\left[k_{2} \sin 2 \phi+k_{3} \cos 2 \phi\right]
\end{aligned}
$$

in which the constant $R$ is equal to $h \tan \theta$, where $h$ is the height of the airglow layer $(\sim 240 \mathrm{~km})$, and $k_{1}, k_{2}$, and $k_{3}$ are defined as

$k_{1}=\frac{\partial u}{\partial x}+\frac{\partial v}{\partial y}$ $k_{2}=\frac{\partial u}{\partial y}+\frac{\partial v}{\partial x}$

$k_{3}=\frac{\partial v}{\partial y}-\frac{\partial u}{\partial x}$

in which $k_{1}$ is the divergence of the flow. Thus, the procedure for processing the data is to fit the set of 8 azimuthal line-of-sight speeds to a second-order Fourier series with coefficients that may be identified with the 5 parameters of Eq. (3), namely $u_{o}, v_{o}, k_{1}, k_{2}$, and $k_{3}$. However, there are six unknowns associated with the parameters in the Taylor series expansion of the neutral wind components $u$ and $v$ in Eqs. (1a) and (1b), and so we lack a sixth equation required for the complete determination of these unknowns from the measurements.

We note that the vorticity of the flow is given by

$\xi=\frac{\partial v}{\partial x}-\frac{\partial u}{\partial y}=\left(\frac{2}{\varepsilon} \frac{\partial v}{\partial t}-k_{2}\right)$

where we have introduced the assumption that the spatial derivative, $\frac{\partial v}{\partial x}$, can be replaced by a temporal derivative, given that $x=\varepsilon t$, where $\varepsilon$ is the tangential speed of the Earth at the latitude of the FPI observatory. The vorticity parameter cannot be measured by a single FPI observatory, because only radial line-of-sight measurements can be made of the wind vector; thus, the gradient $\frac{\partial v}{\partial x}$ cannot be determined, because the rate of change of the wind speed in the direction perpendicular to the line-of-sight cannot be measured.

However, if the time dependence of the wind field is dominated by the local time variation, as argued by Burnside et al. (1981), then the temporal rate of change can be substituted for the spatial derivative. This assumption should apply to the low-latitude wind field, as the data indicate that the time rate of change for either wind component, which is dominated by the local-time dependence of the underlying tidal winds, is relatively slow compared to the cycle interval of $30 \mathrm{~min}$. With this assumption, a linear set of equations may be solved to extract the mean zonal and meridional winds, $u_{0}$ and $v_{0}$, and the four partial derivatives of wind speed with respect to the zonal and meridional directions.

Burnside et al. (1981) claimed that an estimate of the vertical wind can be inferred from the determination of the horizontal divergence of the flow. With the two assumptions of an isothermal atmosphere at the level of the 630-nm airglow layer and no variation of the horizontal velocity components with height (due to the high viscosity of the thermosphere), Burnside et al. (1981) showed that the horizontal wind divergence $\nabla \bullet \boldsymbol{U}$ is related to the vertical wind velocity by the equation:

$\nabla \bullet \boldsymbol{U}=-\frac{\partial w}{\partial z}$

which Burnside et al. (1981) showed may be approximated as

$w=H\left(\frac{\partial u}{\partial x}+\frac{\partial v}{\partial y}\right)=H k_{1}$ 
where $H=k T / m g$ is the atmospheric height scale, which is $\sim 50 \mathrm{~km}$, as long as there is no significant change of pressure with time. This approach, as noted by Burnside et al. (1981), produces an estimate for the vertical wind $w$ with an uncertainty that is $\sim 10$ times less than the uncertainty in direct Doppler shift determinations, $\sim 5 \mathrm{~ms}^{-1}$. We will discuss the validity of this determination of the vertical wind using this assumption and the results obtained and presented in this paper.

\section{Results for the new observing mode}

The measurements obtained thus far with this new observing mode ( 8 azimuthal directions and the zenith) include $\sim 20$ nights for 2005 and 2006. Figure 4 illustrates the quality of the wind, temperature, and intensity data obtained for the observations of 3-4 August 2005, for all nine directions, using an exposure time of $120 \mathrm{~s}$ for each image obtained. The uncertainties in these results for wind and temperature are $7-8 \mathrm{~ms}^{-1}$ and $25-30 \mathrm{~K}$, respectively. Occasionally, there is some disagreement between the results of the two fringes for periods of weak signals. Consequently, for the preparation of wind field maps as described below, we use the results obtained for the innermost fringe marked by the black lines. The results for this inner order should not be affected by the possible under-sampling for the outer annular rings that might exist when the 630-nm intensity is weak.

The temperature data for the 3-4 August 2005, observations plotted in Fig. 4, shows an overall nocturnal cooling throughout the night, starting with typical temperatures of $\sim 800-850 \mathrm{~K}$ at 01:00 UT and decreasing to $\sim 650 \mathrm{~K}$ by 09:00 UT in several directions. (Local midnight is 05:00 UT). The mean nighttime temperature is within the range of 750-800 K, consistent with expected thermospheric temperatures at solar minimum. Inspection of the temperature data between 06:00 and 08:00 UT (01:00 to 03:00 LT) shows the appearance of a strong MTM event with an amplitude of $\sim 150 \mathrm{~K}$. The amplitude is greater for the north set of points compared to the south direction. It is not surprising that for winter observations the MTM event might occur as late as 07:00 UT (02:00 LT). The statistical study of Herrero and Spencer (1982) found the time for the MTM appearance was $\sim$ 04:00 LT for the winter hemisphere at $16^{\circ} \mathrm{S}$.

Figure 4 results for the south direction show the development of a northward meridional wind flow of 50 to $75 \mathrm{~ms}^{-1}$ at 06:00 UT. The zonal wind is eastward as expected, but there is seen first an increase of the speed from 50 to $150 \mathrm{~ms}^{-1}$ in the evening hours reaching a maximum near 04:00 UT in the west direction.

Figure 5 illustrates the Fourier decomposition of the lineof-sight winds for each of the 16 cycles obtained on the night of 30-31 August 2005. The black line going through the data points corresponds to the variation of the line-of-sight (los) wind speed with azimuth angle as modeled by the sum of the
Fourier harmonic terms. As expected, the variation of the wind speed with the azimuth angle shows a positive maximum near $90^{\circ}$ (i.e. the eastward zonal wind) of $\left(\sim 100 \mathrm{~ms}^{-1}\right)$ and the meridional component (angles of $0^{\circ}$ or $180^{\circ}$ ) tends to be weak.

Figure 6 shows plots of the 630-nm intensity (averaged for all directions for each $15 \mathrm{~min}$ period) and the Fourier decomposition analysis results obtained for three nights of 25-26 August, 26-27 August, and 30-31 August in 2005. Each night exhibits an enhancement in the 630-nm intensities along with a MTM event of $100-150 \mathrm{~K}$ in amplitude. The time of the MTM appearance for the first two nights is near midnight (05:00 UT) but is later, near 06:00-07:00 UT, for the third night. The second, third, and fourth rows of this figure show plots of the temporal variations of the mean meridional speed $\left(v_{0}\right)$, the mean zonal speed $\left(u_{0}\right)$, and the temperature averaged over all ten directions in each cycle. The values for $u_{0}$ and $v_{0}$ have been multiplied by the factor of $1 / \sin \theta$ to convert from line-of-sight to horizontal winds. Also shown in the temperature plot is a plot of model temperature values (black line) obtained using the NRL-MSIS00 model for each of the three nights calculated using the $F_{10.7}$ solar flux intensity and the magnetic activity for each case.

The mean winds, $u_{0}$ and $v_{0}$, and the four gradients of the horizontal winds $u$ and $v$ with respect to the zonal and meridional directions, $x$ and $y$, were calculated following the analysis procedure described in Sect. 4. These values were used to determine the temporal variations of divergence, vorticity, and the wind vectors between $-12^{\circ}$ and $-20^{\circ}$ latitudes for each of the three nights in Fig. 6 , at $2^{\circ}$ intervals for latitude and $30 \mathrm{~min}$ for time. The bottom row of panels in Fig. 6 shows plots of the residual temperatures (Tdata minus TMSIS) and the inferred vertical wind computed using Eq. (7).

The vertical wind behavior depicted exhibits small vertical flows for the 25-26 August night, an upward surge peaking at $\sim 5-6 \mathrm{~ms}^{-1}$ from 04:30 to 06:00 UT for the 26-27 August night, and an extended period of downward flow of 5 to $10 \mathrm{~ms}^{-1}$ from 04:00 to 06:00 UT for the 30-31 August 2005 night. Thus, there is no consistent behavior of the inferred vertical wind relative to the development of the MTM peak. The first two nights show the peak upward vertical wind occurring just after the MTM peak, while for the third night, the inferred vertical wind shows a sustained period of downward flow prior to the development of the MTM peak.

The plots of the calculated wind field vectors for the latitude range of $-12^{\circ}$ to $-20^{\circ}$ and for the time period of 01:00 to 08:00 UT (local midnight is 05:00 UT) are presented in Fig. 7 for these three August nights. These vectors are calculated using the equations (Eq. 1a, 1b) evaluated as described in Sect. 4. The time derivatives of the zonal wind for the east and west directions were determined, and the mean used for this calculation. The derivative was determined from the ratio of the velocity difference to the time difference for successive cycles. Since the averaged zonal wind speed, $u_{0}$, varies by only a few $\mathrm{ms}^{-1}$ over $30 \mathrm{~min}$, the error represented by this 


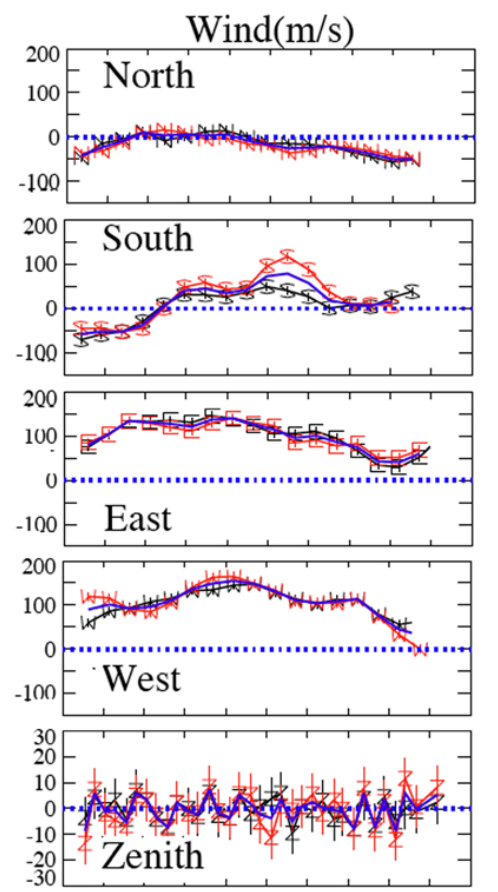

\section{3-4 August 2005}
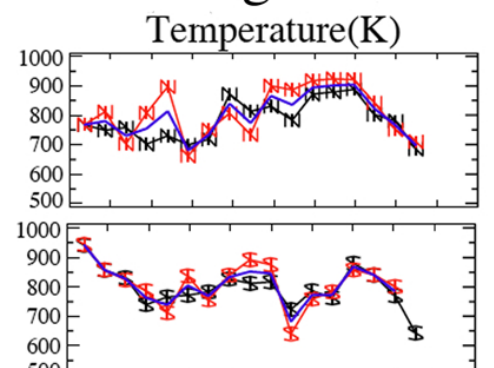

$$
500
$$

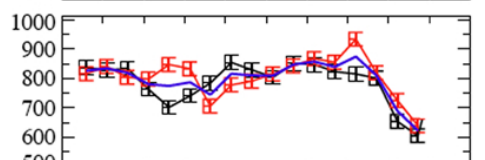$$
1000
$$

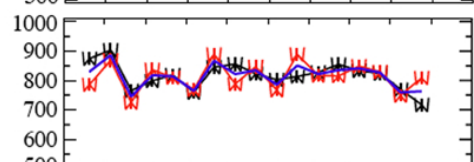

$$
500
$$
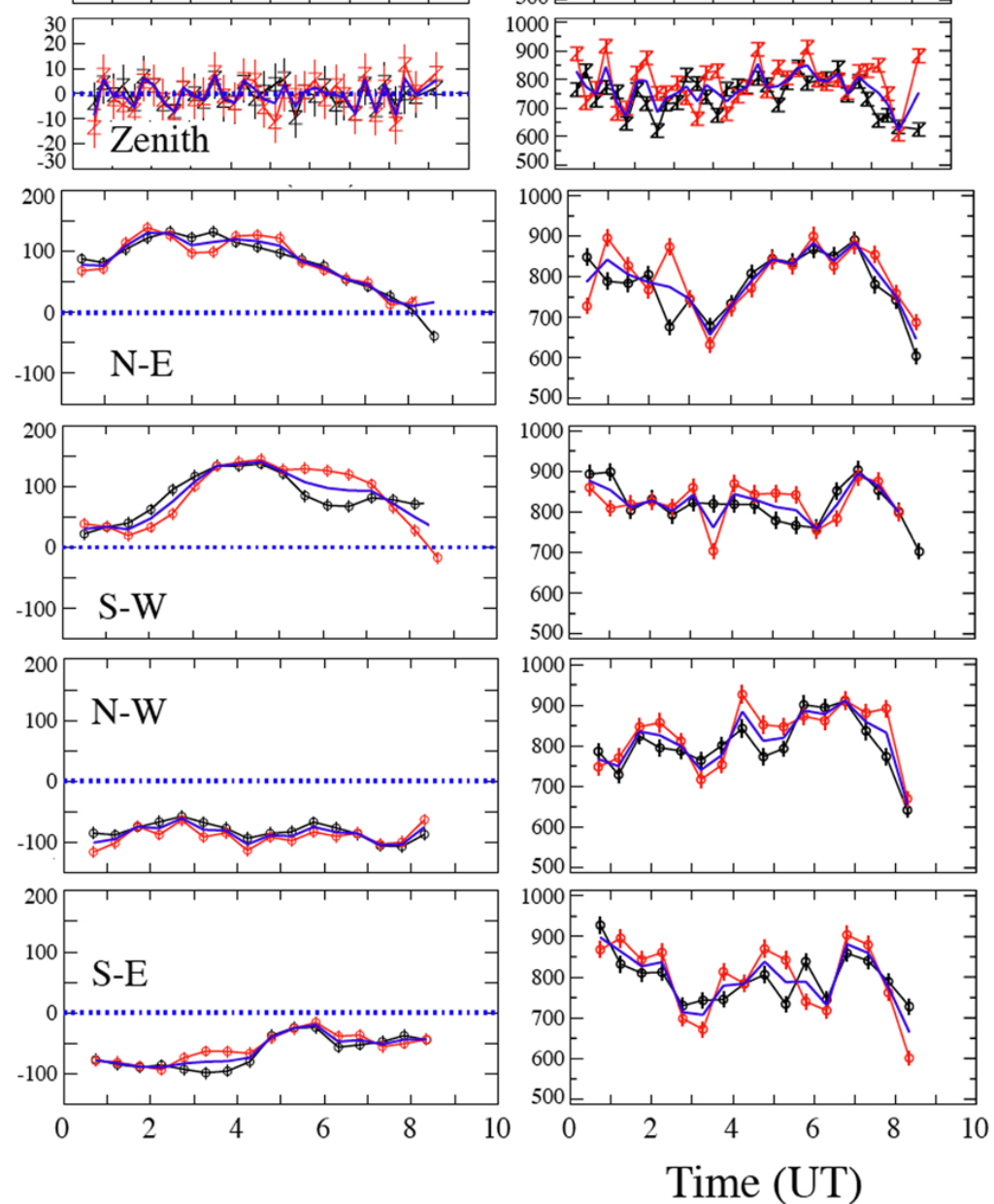
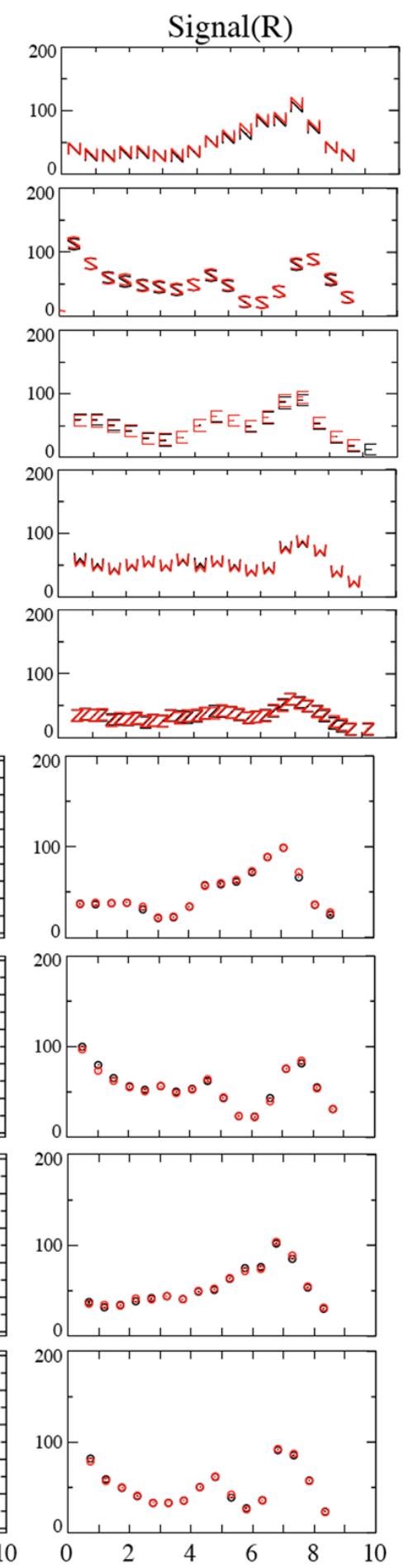

Fig. 4. Arequipa FPI measurements of horizontal wind components (left column), temperatures (middle column) and 630-nm intensities (right column) for 3-4 August 2005. The exposure time for each direction was $120 \mathrm{~s}$. The red, black, and blue lines in each panel are plots for the inner and outer orders observed in each image and the average of these results, respectively. 
30-31 August 2005
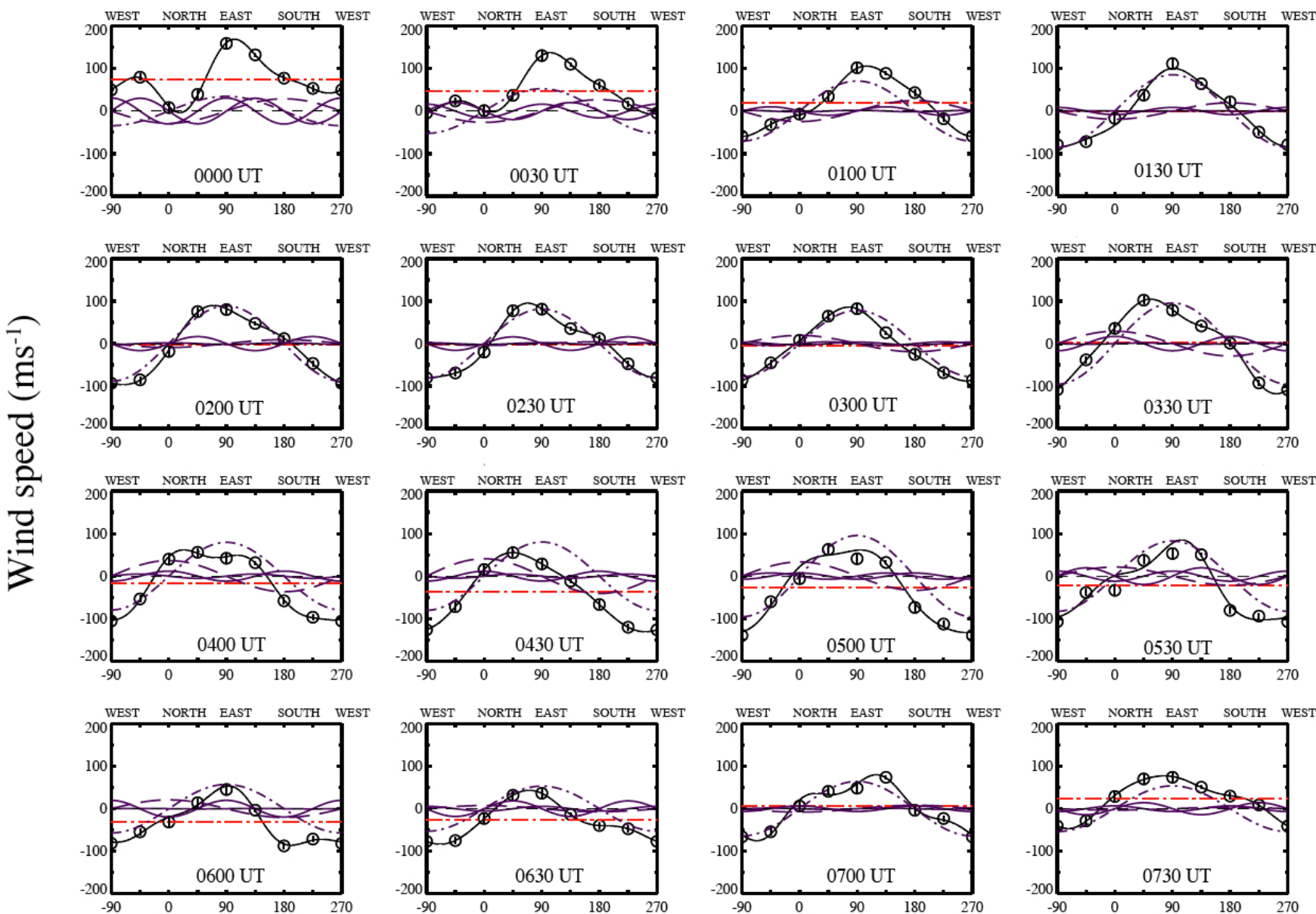

Azimuth $\left({ }^{\circ}\right)$

Fig. 5. Line-of-sight neutral wind speeds (round data points) observed on 30-31 August 2005, plotted against azimuth angle for 16 observing cycles from 00:00 UT to 07:30 UT. The vertical line within each data point indicates $\pm 1 \sigma$ standard deviation. Each observing cycle includes 8 azimuthal line-of-sight directions distributed between $0^{\circ}$ and $360^{\circ}$ at $45^{\circ}$ intervals. The contributions from the three harmonics for the Fourier decomposition (Eq. 3) of the neutral wind field are shown as dashed (first order or second order) or solid (third order) lines. The black line is the overall fit using these three harmonics. The dotted red line is the divergence calculated from the gradients determined from this analysis.

means of determining the derivative necessary to evaluate the vorticity as represented by Eq. (5b) should be small.

For each night the wind field plots show the development of a northward (equatorward) surge of $25-75 \mathrm{~ms}^{-1}$ prior to the onset of the MTM event. In the case of 25-26 August, the northward flow is seen extending from 02:00 UT to $05: 15$ UT, with the peak of the surge seen at 03:30-03:45 UT, $\sim 1.5-2.0 \mathrm{~h}$ prior to the peak of the MTM event seen at 05:30-05:45 UT. The magnitude of the surge is perhaps 25$30 \mathrm{~ms}^{-1}$, and the amplitude of the MTM is $\sim 200 \mathrm{~K}$.

For the following night of 26-27 August 2005, the northward flow appears between 01:30 and 04:00 UT, with the peak at 02:15 UT, and the MTM peak is seen at 03:4504:15 UT, a difference of $\sim 2.0 \mathrm{~h}$. The meridional speed of the surge in this case was $50-60 \mathrm{~ms}^{-1}$ and the MTM amplitude was $\sim 150-160 \mathrm{~K}$. Finally, for the third night, 3031 August 2005, the development of the northward meridional flow with a peak speed of $\sim 50-75 \mathrm{~ms}^{-1}$ took place between 03:30 UT and 04:30 UT, and the appearance of the MTM with an amplitude of $\sim 100 \mathrm{~K}$ was delayed to 06:0006:15 UT, a difference of $\sim 2.0 \mathrm{~h}$ from the peak of the wind northward surge and the MTM enhancement.

In each of these three cases, the subsequent decrease of the MTM after the peak was associated with an interval of 


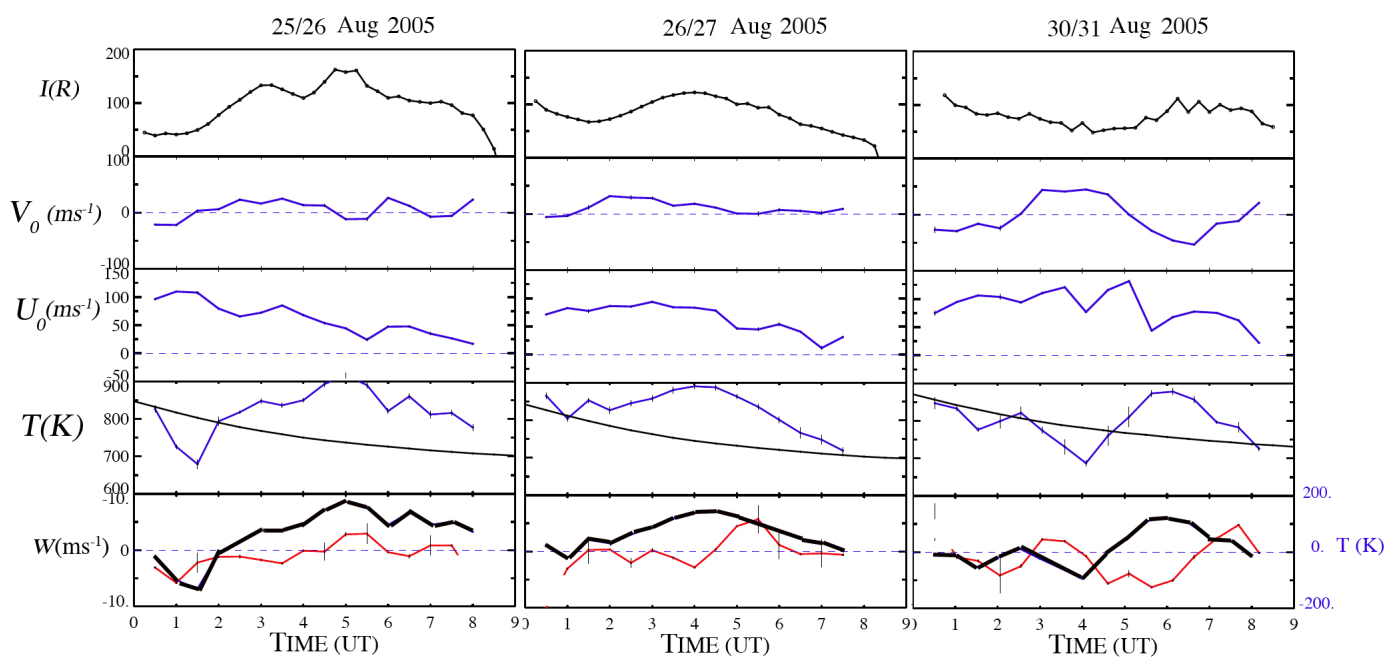

Fig. 6. Averaged intensities and Fourier decomposition analysis results for three nights in late August, 2005. Temporal variations of the averaged 630-nm intensity (all directions) and the computed mean speeds for the horizontal meridional (V0) and zonal (U0) wind components are plotted in the top three rows, respectively. The time sequence of cycle-averaged temperatures (blue data points and line) and the predicted values computed with the NRL-MSIS00 model (black line) are plotted in the fourth row. The red and black lines in the bottom row show the temporal variations of the vertical wind $w$ and the temperature difference (FPI data minus the computed MSIS values), respectively. Local midnight is 05:00 UT. The vertical black line for each data point indicates the range of $\pm 1 \sigma$ standard deviation.

meridional wind reduction or reversal to poleward that took place over an $1-2 \mathrm{~h}$ interval. For the first night, in the north near $-12^{\circ}$ to $-14^{\circ}$, the wind speed is weak for $1.5-2.0 \mathrm{~h}$ after the MTM peak. Farther to the south, it can be seen that there is an increase in the poleward direction of $25-35 \mathrm{~ms}^{-1}$. A similar behavior can be seen for the other two nights. For the third night, 30-31 August, the reversal can be seen quite clearly, especially in the region of $-12^{\circ}$ to $-16^{\circ}$ between 05:45 and 06:15 UT. This behavior for all three nights suggests a weak reversal of the meridional wind flow from equatorward to poleward occurring after the MTM peaks.

For three nights near the spring and fall Southern Hemisphere equinoxes Fig. 8 illustrates the time behavior of the mean zonal and meridional wind, as well as the observed and the calculated MSIS00 temperatures. Figure 9 shows the corresponding thermospheric wind flow patterns for these three nights. For each, in Fig. 9, a strongly-developed equatorward flow is seen, with the northward wind reaching maximum speeds of $35 \mathrm{~ms}^{-1}, 100-125 \mathrm{~ms}^{-1}$ and $50-65 \mathrm{~ms}^{-1}$ at 01:30-02:00 UT, 04:15-04:30 UT and 05:00-06:00 UT, respectively. For the early September night, the delay between the northward surge at 02:00 UT and the MTM peak at $05: 30 \mathrm{UT}$ is about $3.5 \mathrm{~h}$. For this night a period of poleward wind between 04:00 and 06:00 UT associated with the MTM appearance is evident, with speeds of $\sim 50 \mathrm{~ms}^{-1}$. For the last two nights the difference in time between the peak of the equatorward meridional flow and the peak of the MTM enhancement, $\sim 100-125 \mathrm{~K}$, is about $30 \mathrm{~min}$, less than the $\sim 2 \mathrm{~h}$ time difference seen for the three cases presented in Fig. 7.
For the 29-30 September night, there is a clear abatement in the meridional wind flow after the MTM passage.

Again, in Fig. 8 there is no clear correlation in the behavior of the inferred vertical wind and the MTM peak. For the first night, the vertical wind is downward at $3-5 \mathrm{~ms}^{-1}$ during the MTM peak. In the second night, $w$ varies in a sinusoidal way, being downward a few $\mathrm{ms}^{-1}$ an hour prior to the arrival of the MTM peak, and then reversing to upward flow as the MTM passes. For the third night, there is an upward flow of $2 \mathrm{~ms}^{-1}$, peaking just prior to the MTM peak.

Figure 10 presents the temporal variations of the averaged temperatures and the MSIS model, together with the maps of the horizontal wind vector field for the two nights of 1011 June 2005, and 7-8 July 2005. For these two examples, the MTM peak is not evident. For both cases, the temporal variations of the observed temperatures agree well with that predicted by the NRL-MSIS00 model predictions. Here, for these two examples the wind field maps show no indication of a meridional wind flow toward the equator, unlike that noted for the six nights of Figs. 6 and 8. In the early evening hours for both nights the wind is toward the southeast in accord with the nominal seasonal behavior (Biondi et al., 1999), and then after 02:00 UT the flow is dominated by the eastward component.

The two maps in Fig. 10 illustrate the typical velocity field that is dominated by the day-to-night pressure gradient, which would drive eastward winds during the night, combined with an early evening poleward component (southward) that is a result of the cross-hemispheric flow from 


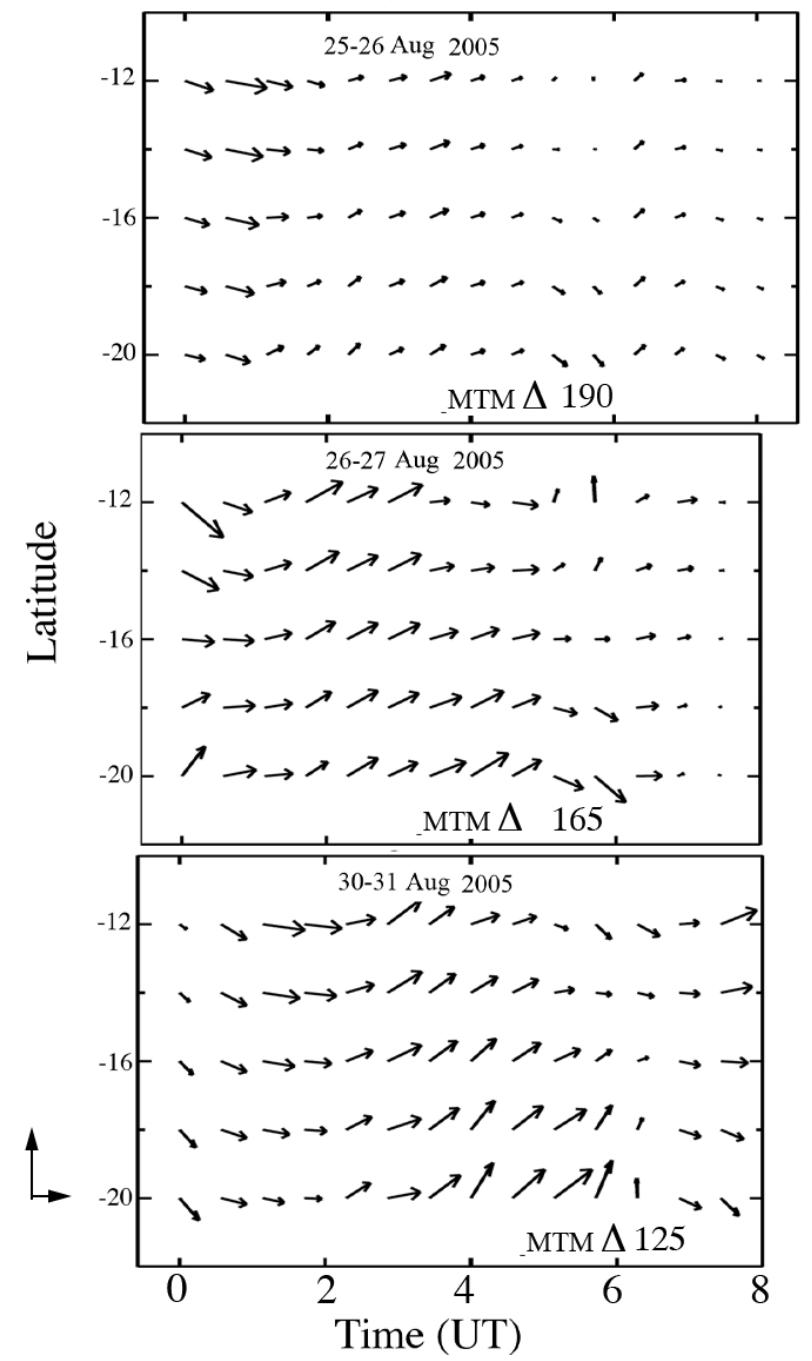

Fig. 7. Horizontal wind maps for three nights in August, 2005. Neutral wind vectors are computed for $2^{\circ}$ intervals of latitude between $-12^{\circ}$ and $-20^{\circ}$ and every $30 \mathrm{~min}$ in UT. Local midnight occurs at 05:00 UT. The time of the MTM peak amplitude is indicated by the open triangle next to the MTM label and the amplitude of the MTM peak is indicated. An horizonal meridional or zonal wind speed of $100 \mathrm{~ms}^{-1}$ is represented by the scale distance of $2^{\circ}$ latitude or $30 \mathrm{~min}$, respectively (see insert, lower left corner).

summer to winter. The speed of this poleward component diminishes during the hours prior to midnight. These two wind patterns represent examples of the nominal behavior of the low-latitude thermosphere dynamics for weak tidal forcing when there is no detectable MTM event.

Comparison of the behavior seen in Fig. 10 relative to those of Figs. 7 and 9 shows that the MTM event and the underlying tidal forcing modified significantly the background flow. The highlight of the wind patterns for the six nights of Figs. 7 and 9 is the appearance of a wind surge between 03:00 and 06:00 UT toward the equator, with considerably higher speeds seen for this meridional flow on the two nights of 29-30 September 2005, and 5-6 April 2006 (Fig. 9). For the observations on 30-31 August, a boundary developed between poleward winds (lower portion of the figure) and equatorward winds (upper portion) near $-16^{\circ}$ latitude and 06:00 UT.

Figure 11 illustrates the night-to-night variability seen in these wind field maps for observations obtained over five nights in July, 4-5 July to 8-9 July, and 28-29 July. (This figure includes the results for 7-8 July already shown in Fig. 11.) The MTM label inserted in each figure marks the time when the MTM peak was seen for each night. MTM events were seen for these nights, with amplitudes of $\sim 75 \mathrm{~K}$, $\sim 90 \mathrm{~K}, \sim 75 \mathrm{~K}, \sim 0 \mathrm{~K}, \sim 100 \mathrm{~K}$, and $\sim 100 \mathrm{~K}$, respectively. For these nights, the cross-hemispherical flow (directed to the southeast) is seen in the early evening from 00:00 to 03:00 UT, followed by the appearance of equatorward winds during the midnight and early morning hours of 04:00 to 09:00 UT. The late appearances of the MTM are in accord with what is expected from the climatology study of Faivre et al. (2006) and Herrero et al. (1993). Inspection of these July nights shows indications of the development of anticyclonic flow (clockwise). For example, for 6-7 July, the direction of the wind vector shifts clockwise from northward (at 02:00 UT) to southward (at 06:00 UT).

\section{Discussion}

Data for 13 nights from the 2005-2006 Arequipa FPI observations were obtained using the new observing strategy featuring eight sequential azimuthal positions from $0^{\circ}$ to $360^{\circ}$. The analysis of these results has revealed new information regarding the behavior of the equatorial thermospheric winds and temperatures during solar minimum regarding the formation of the MTM peak. Because the 630-nm airglow intensities seen were $\sim 5$ times weaker than for solar maximum, observing the thermospheric dynamics was previously a difficult challenge for this phase of the solar cycle. As an illustration of how much improved these results are, we note that the FPI Arequipa results reported by Colerico et al. (1996) for observations obtained in October 1993, show an acquisition rate of $\sim 1-2$ data point per hour per direction with uncertainties of $15 \mathrm{~ms}^{-1}$ and $40-45 \mathrm{~K}$. In contrast, the improved Arequipa FPI data presented in Fig. 3 shows a rate of 4-5 data points per hr with uncertainties of $5 \mathrm{~ms}^{-1}$ and $15-$ $20 \mathrm{~K}$. The new results provide new insights into the behavior of the MTM, and we summarize the findings here.

\subsection{Equatorward surge}

Results from 11 nights illustrated the development of the MTM peak is preceded by a meridional flow of air toward the equator, that appears near midnight. This surge of air toward the equator generally reaches a maximum amplitude near midnight (04:00-06:00 UT), and for each of these, 


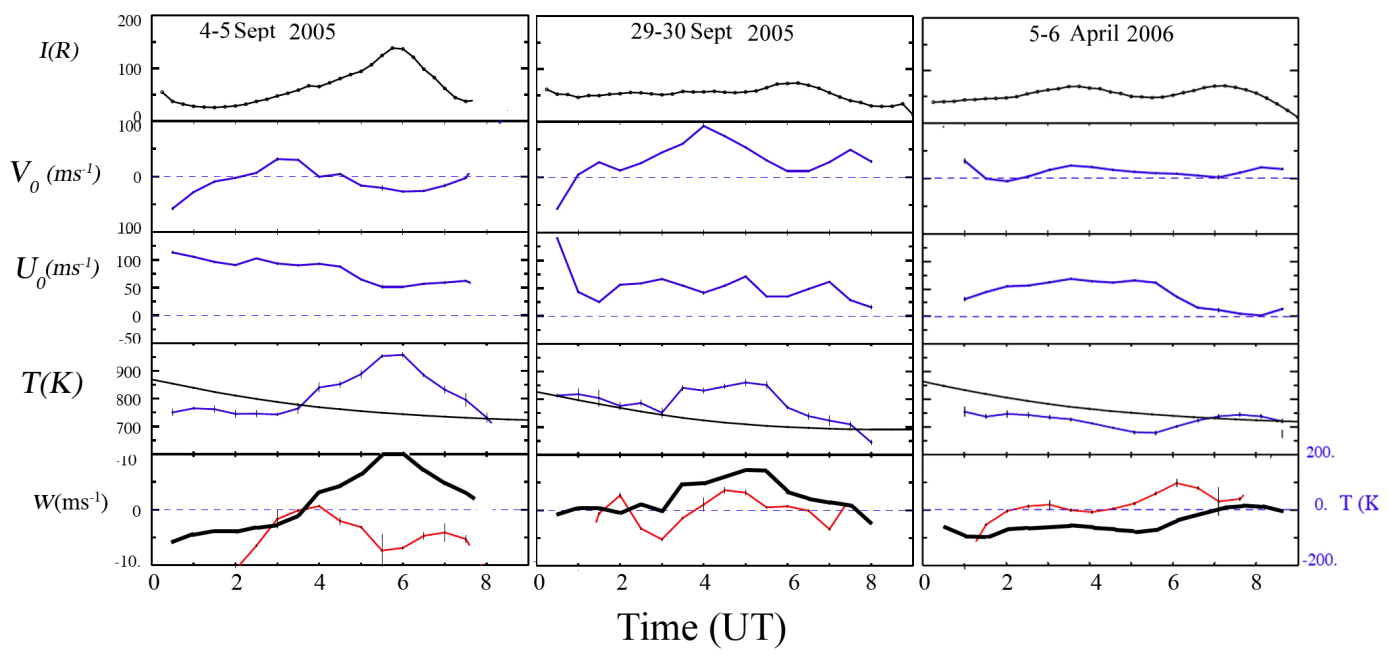

Fig. 8. Results for 3 nights with dates near equinox: September 2005 and early April 2006, respectively. The top three rows show the temporal variations of the averaged $630-\mathrm{nm}$ intensity and mean speeds for the horizontal meridional $\left(v_{0}\right)$ and zonal $\left(u_{0}\right)$ wind components, respectively. The fourth row shows the time sequence of cycle-averaged temperatures (blue data points and line) compared with the NRLMSIS00 model (black line). The bottom row presents temporal variations of the vertical wind (red line) and the temperature difference (black line, FPI data minus NRL-MSIS00 model).

MTM peaks were seen with amplitudes between $100 \mathrm{~K}$ to $200 \mathrm{~K}$ occurring later in local time by 30 to $120 \mathrm{~min}$. In contrast, the two nights of data plotted in Fig. 10 were examples that indicated either the lack of any MTM event, or that the MTM amplitude was weak, $\sim 30 \mathrm{~K}$. For both examples, the meridional northward flow was found to be non-existent or weak. This finding of weak northward meridional winds for nights with weak MTM events was also true for other nights in our database.

The equatorward flow is consistent with that associated with the propagation of tidal waves into the thermosphere region from altitudes below. The modeling work of Fesen (1996) predicts for the Southern Hemisphere during the midnight hours an equatorward meridional flow that is generated by the migrating semi-diurnal 2-2 tidal mode, with an amplitude that is strongest in the summer, somewhat weaker in the winter, and weakest at the equinoxes. The strong equinoctial winds (speeds greater than $75-100 \mathrm{~ms}^{-1}$ ) seen in our results (Fig. 9 and other nights not shown) during both equinoxes, and also predicted by the modeling analysis, therefore suggest that the 2,2 tidal mode is reinforced by the asymmetric 2,3 tidal wave, which is weak in the winter but strongest at this location during the equinoxes (Fesen, 1996). Possibly non-migrating tidal waves are contributing to the development of strong nighttime equatorward winds.

It is also possible that the terdiurnal tidal wave contributes to the production of this surge. Crary and Forbes (1986) found that the observed nighttime behavior of the ionosphere over Arecibo could not be properly modeled unless the terdiurnal tidal wave was included. Colerico et al. (2006) suggested that the MTM amplitude modeled with the TIEGCM was too weak $(\sim 30 \mathrm{~K})$ compared with observations (100$200 \mathrm{~K}$ ), because the TIEGCM prescription for tidal forcing is incomplete without any provision for the terdiurnal component. These conclusions are consistent with Herrero et al. (1993), who determined that within the low-latitude thermosphere the amplitude of the terdiurnal tide was comparable with that of the semi-diurnal tide. However, one should bear in mind that the ion-neutral coupling within the thermosphere, as described by Mayr et al. (1979), involving the nonlinear coupling of the diurnal and semi-diurnal tidal modes caused by the EUV in situ forcing of the thermosphere, may also result in the appearance of a terdiurnal tidal component.

When the MTM peak is weak or non-existent, the meridional surge near midnight is either not present or weak, suggesting that the tidal forcing from below is weak, since this equatorward flow of air represents the southern cell of the meridional tidal circulation. There is also a northern cell of the tidal circulation in the Northern Hemisphere that is moving air toward the equator. The consequential convergence produces a downward flow of air, resulting in the adiabatic heating of the thermosphere and the consequent formation of the MTM structure near the geographic equator. Thus, at equinox a weak tidal circulation would result in no formation of the MTM, or a peak with a small amplitude. The details of the spatial distribution of the MTM heating and its temporal evolution remain unclear, and this will be discussed further below.

The analysis of the observations showed that the local time separation between the peak northward surge of air and the MTM peak temperature decreased from $\sim 2 \mathrm{~h}$ for winter to $\sim 30 \mathrm{~min}$ at the equinox. This is consistent with the 


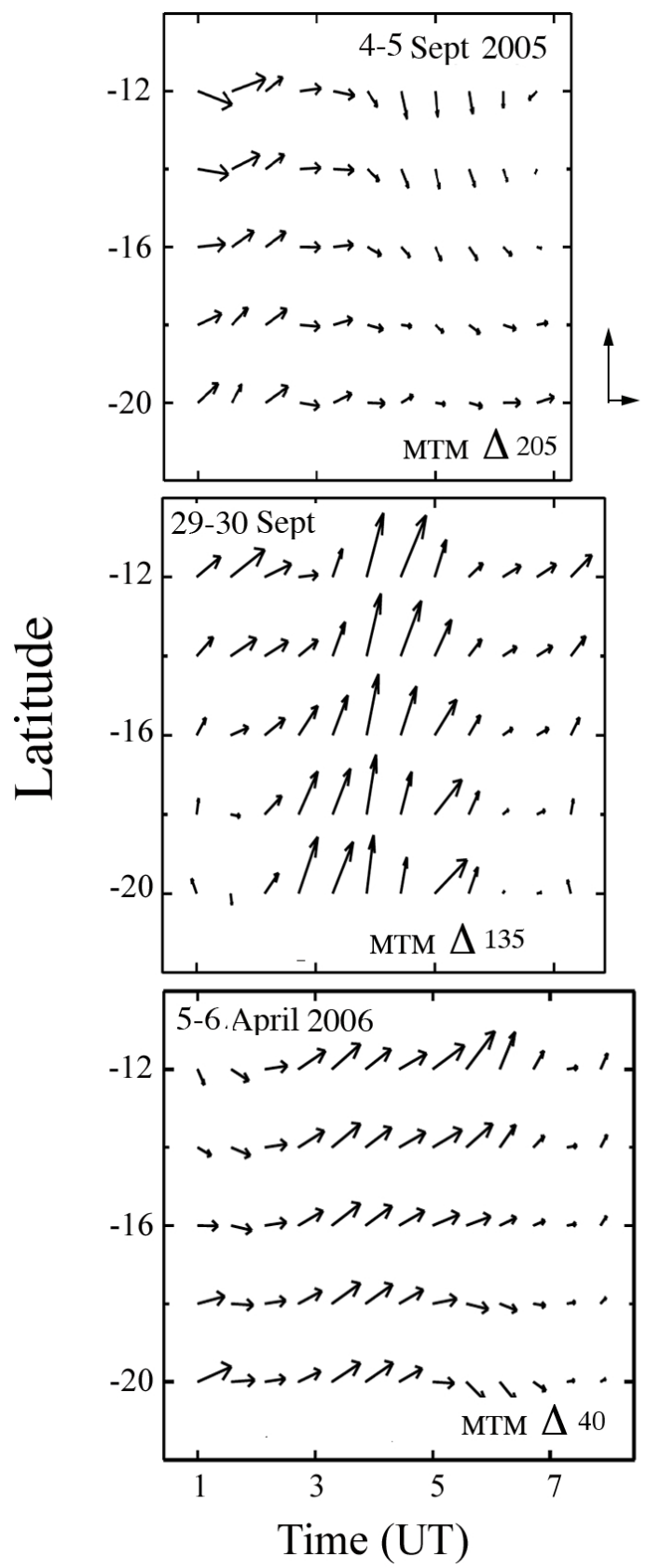

Fig. 9. Horizontal wind maps for the three nights of Fig. 8: 4 5 September 2005, 29-30 September 2005, and 5-6 April 2006. Local midnight occurs at 05:00 UT. The horizonal meridional or zonal wind speed of $100 \mathrm{~ms}^{-1}$ is represented by the distance of $2^{\circ}$ latitude or $30 \mathrm{~min}$, respectively. The time and the magnitude of the MTM peak are indicated by the triangle next to the MTM label and the number, respectively.

location of the MTM compressional heating to the north of the geographical equator during the $\mathrm{SH}$ winter and farther to the south at the equinox. This result is also consistent with the findings reported in the study of the MTM clima- tology (Faivre et al., 2006) that the times of the appearance of the MTM is delayed $2-3 \mathrm{~h}$ for winter nights, compared with equinoctial nights. Thus, in the winter more time is required for the air to move north from Arequipa to contribute to the formation of the pressure bulge, and then, following the formation of the MTM, for the MTM to impact the thermosphere over the Arequipa site.

Colerico et al. (1996) reported the 630-nm brightness wave (BW) structure moved at a meridional speed of $\sim 200$ $250 \mathrm{~ms}^{-1}$ in the poleward direction. We estimate a speed of $\sim 50 \mathrm{~ms}^{-1}$ for the meridional northward surge, as this value is typical of our observations. Thus, a calculation of the distance from Arequipa to the region of the pressure bulge can be made for the two seasons by using the times of $30 \mathrm{~min}$ (equinox) and $120 \mathrm{~min}$ (winter) to represent the sum of the elapsed time for the air to move north and the time for the MTM to move south passing over the same separation. The results imply a separation of only $\sim 1^{\circ}$ for the equinox and $\sim 4^{\circ}$ in the winter. Since AE-E data reported by Herrero et al. (1991) shows the pressure bulge to be localized near the geographic equator for the equinox, it is evident that this calculation is not realistic. What is left out is the likely reduction of the tidal wind speed equatorward as the air reaches the region of the pressure bulge. Also, this calculation should include the time required for the MTM formation and allow for the possible variation of the MTM speed toward high latitudes after this formation has taken place.

\subsection{Conceptual model for the MTM latitudinal-LT distri-} bution

To illustrate what is expected for the geometry of the MTM structure, Fig. 12a presents from the AE-E observations presented by Herrero and Spencer (1982) a reproduction of the contour plot of the latitudinal statistical distribution of thermospheric temperatures at $240 \mathrm{~km}$ plotted against local time. These results were compiled from the observations of many orbits for the period of the Southern Hemisphere winter during moderate solar activity, 1977. The V-shape shaded region indicates the areas when the MTM feature is easily seen against the distribution of background nighttime temperatures. The pressure bulge location is expected to be within the two arms of the MTM structure covering the region near the geographic equator.

Using the same latitude and local time coordinates as that of Fig. 2a, Fig. 12b provides a conceptual diagram that illustrates the location of the pressure bulge. Also plotted is the latitudinal distribution of the MTM plotted along the perimeter of the pressure bulge. The intent of this plot is to illustrate how the MTM may be generated by the convergence of winds from the polar regions and from the dayside. The justification for showing the MTM as a narrow strip along the edge of this zone is the double wing structure seen in the results presented in Herrero and Spencer (1982). Herrero et al. (1993) also noted that when AE-E wind data were examined as a 


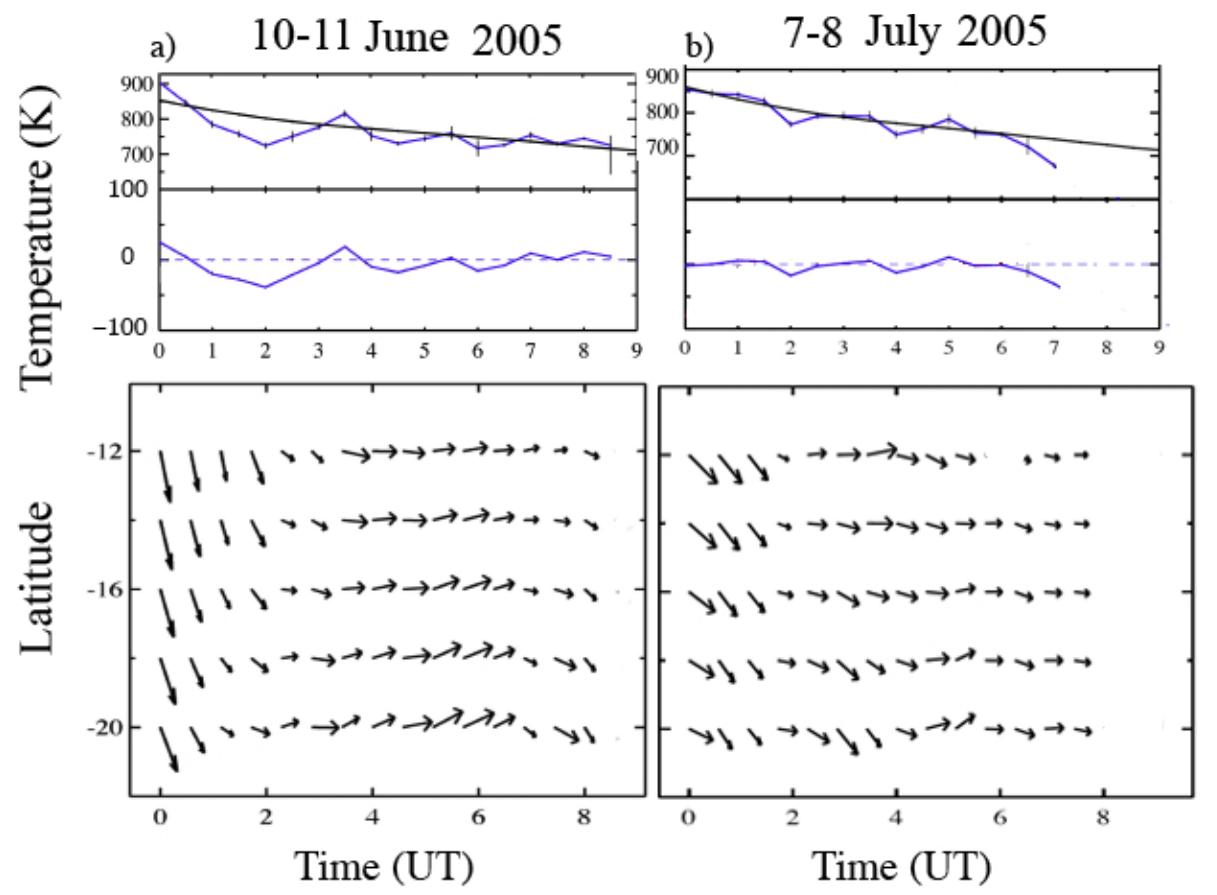

Fig. 10. Results for the nights of (a) 10-11 June 2005, and (b) 7-8 July, 2005. The top row of panels shows the temporal sequence of cycle-averaged temperatures (blue data points and line) compared with the NRL-MSIS00 model (black line). The middle and bottom rows of panels show for both nights the plot of the differences between observed temperatures and MSIS model values and the maps of horizontal winds, respectively. Local midnight is 05:00 UT. The horizonal meridional or zonal wind speed of $100 \mathrm{~ms}^{-1}$ is represented by the distance of $2^{\circ}$ latitude or $30 \mathrm{~min}$, respectively.

function of latitude and local time, nighttime reversals were only seen at latitudes outside the borders of a triangular zone that was identified as the region of the pressure bulge. The apex of this zone started at the equator near 22:00 LT and the latitudinal width of this zone extended to $\pm 15^{\circ}$ for MTM events taking place at 03:00 LT. Within this zone, the AE-E data showed that the meridional wind would be reduced in speed but not reversed in direction.

Evidentally, the MTM formation starts near 22:00 LT, when the zonal air converges with the meridional flow of air from both polar regions. The pressure bulge starts to grow, and the back pressure of air from the central region of the pressure bulge prevents the air from continuing its flow into the postmidnight sector. The resulting convergence of air then creates the MTM phenomenon by compressional heating as the air moves downward to lower altitudes.

An indication of a meridional wind reversal in the flow patterns following the tidal midnight equatorward surge is clearly suggested in observations for one night, 4-5 September, of the cases examined. The reversal appears $\sim 4 \mathrm{~h}$ after the peak of the equatorward surge but coincident with the MTM peak. In the other cases studied, what is more clearly observed is an abatement of the equatorward wind. Since the MTM events of Figs. 8 and 9 for 29-30 September 2005, and 5-6 April 2006 were seen between 05:00 and 06:00 UT, it is therefore not surprising that the observed behavior of the meridional wind for these nights is more consistent with an abatement rather than a reversal. For the MTM event on 4-5 September, which took place at 06:00 UT (01:00 LT), the observed reversal would be expected, as the AE-E results (Herrero et al., 1993) show the latitude of Arequipa is located just outside of this pressure bulge zone for this time.

The MTM TIEGCM modeling work described by Colerico et al. (2006) produced no meridional wind reversal, and this was regarded as a failure of the model, possibly due to the omission of the terdiurnal tidal mode not specified as part of the lower boundary forcing. Our results show that an abatement of the meridional wind flow at the time of the MTM peak is more typical of the winter equatorial thermospheric dynamics than a reversal, for these solar minimum MTM observations. The Arequipa FPI data show that the beginning of the abatement coincides with the time of the MTM peak temperature. A reversal generally follows 1 or $2 \mathrm{~h}$ later, not at a time that is coincident with the MTM peak passing (except for the one case of 4-5 September 2005).

Comparing the 630-nm intensity results for the two September nights in Fig. 8 is also of interest. The meridional winds for the 29-30 September night exhibit an abatement, and the 630-nm nightglow MTM enhancement is a factor of $\sim 3$ weaker than for the 4-5 September night, for 


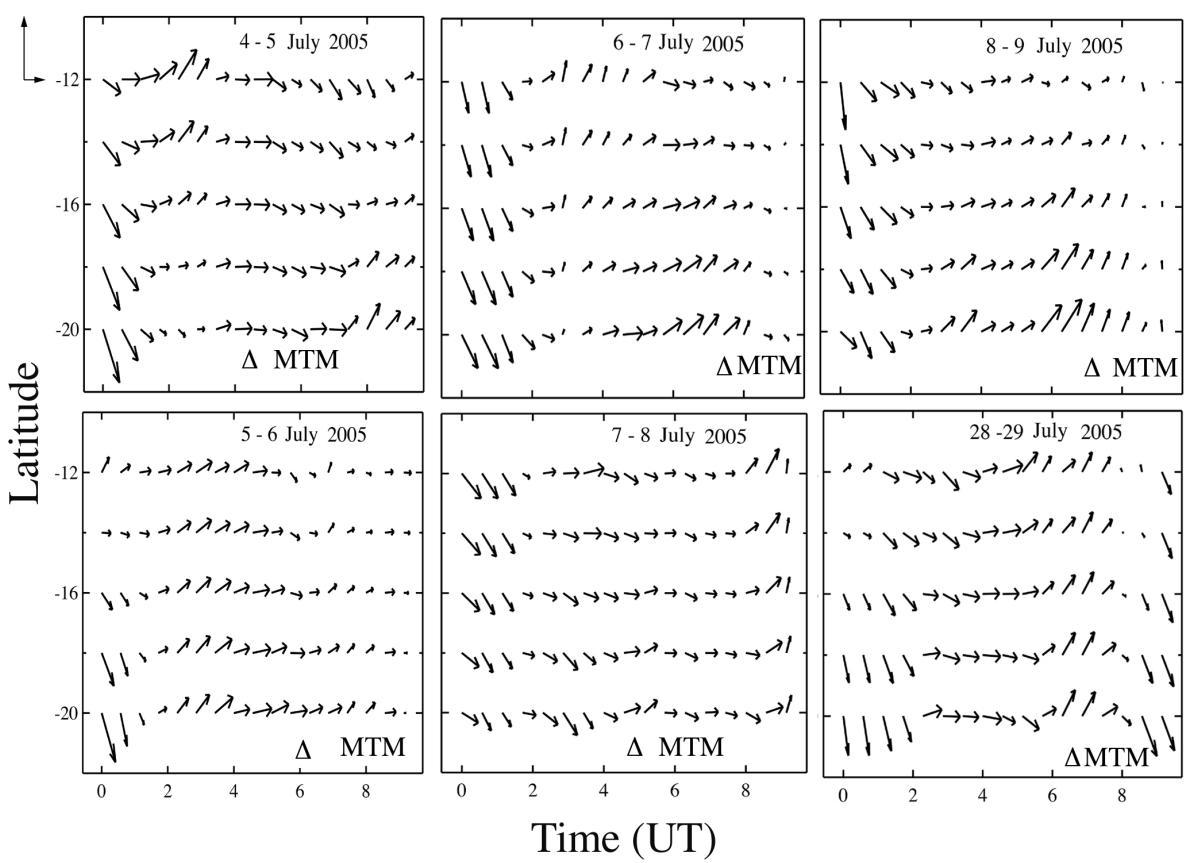

Fig. 11. Horizontal wind maps for six nights of observations in July 2005. Neutral wind vectors are computed for $2^{\circ}$ intervals of latitude between $-12^{\circ}$ and $-20^{\circ}$ and every $30 \mathrm{~min}$ in UT. Local midnight occurs at 05:00 UT. The horizonal meridional or zonal wind speed of $100 \mathrm{~ms}^{-1}$ is represented by the distance of $2^{\circ}$ latitude or $30 \mathrm{~min}$, respectively; a scale insert is shown in the upper left corner. The UT position of the maximum of the MTM event is indicated by the triangle next to the MTM label; the amplitudes are $75 \mathrm{~K}, 90 \mathrm{~K}, 75 \mathrm{~K}, 0 \mathrm{~K}$, $100 \mathrm{~K}$, and $100 \mathrm{~K}$, respectively, for these six nights.

which a reversal is seen for the meridional wind. That the 630-nm MTM intensity peak intensity at 06:15 UT is weaker for the 29-30 September night is consistent with an abatement rather than a reversal in the meridional wind, since less F-region plasma is transported to lower altitudes if the wind component parallel with the flux tube becomes weaker rather than reverses. This behavior of a relatively strong MTM event but a weak 630-nm BW and an abatement in the meridional wind is also seen for the 5-6 April 2006 night, for which the timing of the MTM event, 07:00 UT, is well after local midnight.

\subsection{NCAR-TIEGCM modeling of the MTM phenomenon}

Colerico et al. (2006) noted that the lack of a reversal signature in the modeling results together with the weak MTM amplitude points to a need to include the terdiurnal tidal wave as part of the TIEGCM prescription for the tidal forcing at the lower boundary of the model. The modeling analysis has thus far been constrained to consider only the diurnal and semi-diurnal tidal modes. However, as previously mentioned, the tidal spectral analysis of AE-E data suggested that the contribution from the terdiurnal tidal mode is important (Herrero et al., 1983, 1993), especially during winter, for which the amplitude is comparable with that of the semi-diurnal tide.
As suggested by Mayr et al. (1979), the ion-neutral coupling between the diurnal variation of the F-region plasma density and the semi-diurnal tidal winds may result in the higher order tidal modes being generated. Thus, it may not be necessary to explicitly specify a terdiurnal tidal component from the lower atmosphere in the model if the terdiurnal tide from the ion-neutral momentum coupling dominates. Because of the non-linear coupling between plasma density and the diurnal and semi-diurnal tides, it would be expected that the strength of this particular terdiurnal tidal wave would be variable from one night to the next. The anti-cyclonic behavior in the wind maps presented in Fig. 12 showing equatorward flow followed by a rotation poleward within $\sim 4 \mathrm{~h}$ is consistent with the behavior of a terdiurnal wave, and may suggest that the terdiurnal mode is playing a more significant role in equatorial thermospheric dynamics than previously appreciated.

Figure 13 shows four nights in August 2005 modeled by the NCAR TIME-GCM at the location of Arequipa. The model includes terdiurnal tides forced by the non-linear interaction of the diurnal and semidiurnal tides. One sees that there is no significant MTM feature on any of these nights. In contrast, inspection of the observations presented in Figs. 6 and 7 shows MTM signatures of $\sim 175 \mathrm{~K}, \sim 125 \mathrm{~K}$, and $\sim 100 \mathrm{~K}$, respectively, for the nights, 25-26 August, 26-27 August, and 27-28 August in 2005. Moreover, examination 
of the appearance of this structure from one night to the next suggests that its timing is quite variable from night to night. This suggests a link of the variability of the equatorial tidal structure to the variability of the development of plasma instabilities.

\subsection{Slow local time variation of the zonal wind speed}

The slow decrease of the zonal wind with local time justifies the assumption made regarding the calculations of the four derivatives that appear in Eq. (1). Another way of understanding the assumption, as described by Meriwether and Shih (1987), is to consider that two line-of-sight measurements at two different local times toward a region of the thermosphere are necessary to compose a vector, assuming a zero vertical wind. Thus, by shifting the local-time origin of one or the other of the two series of measurements in local time, any line-of-sight Doppler shift measurement can be combined with another Doppler shift measurement obtained at the same local time to compose a vector determination of the wind speed and direction. In essence, this approach is invoking the same assumption that the longitudinal direction is equivalent to local time.

\subsection{Inferred vertical wind not physically realistic}

Unlike the results reported by Burnside et al. (1981), no systematic correlation of the behavior of the inferred vertical wind with the MTM structure was observed, suggesting that the inferred wind is not physical but represents a failure of one or more of the three assumptions that underlie Eq. (7), which are: the temporal change of the pressure is zero, the vertical wind is constant with height, and the horizontal wind is constant with height. The fact that a $630-\mathrm{nm}$ nightglow enhancement is seen over Arequipa that is generally correlated with the MTM peak does suggest that a vertical wind flow exists shifting plasma to lower altitudes along the magnetic flux tubes and thereby producing increased dissociative recombination and increased 630-nm nightglow intensities.

All-sky imaging observations (Colerico et al., 1996) show from the width of the 630-nm airglow enhancement (BW) that the spatial extent of the MTM phenomenon is about 400$500 \mathrm{~km}$. For a horizontal direction through a cross-sectional cut of the BW, this implies that the vertical wind changes from zero to a downward speed of $\sim 1-2 \mathrm{~ms}^{-1}$ to zero within this distance. A downward speed of this magnitude was estimated by Fesen (1996) to be required to produce a MTM of $30 \mathrm{~K}$. The corresponding time interval for this change is 45$60 \mathrm{~min}$. Because the MTM peak coincides reasonably well in time with the 630-nm enhancement in Fig. 4, this suggests that the heating is localized to within this spatial distribution of the feaure. Consequently, for the typical MTM seen at solar minimum, i.e. with amplitudes as much as 150 to $200 \mathrm{~K}$, it seems unreasonable to assume that the temporal variation in pressure would be negligible. Burnside et al. (1981) esti-

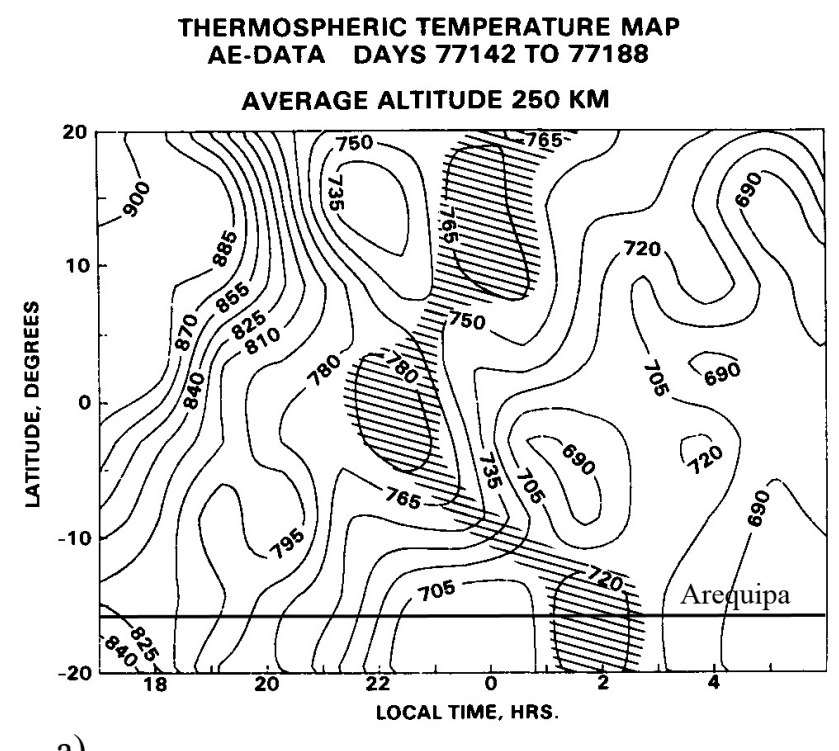

a)

b)

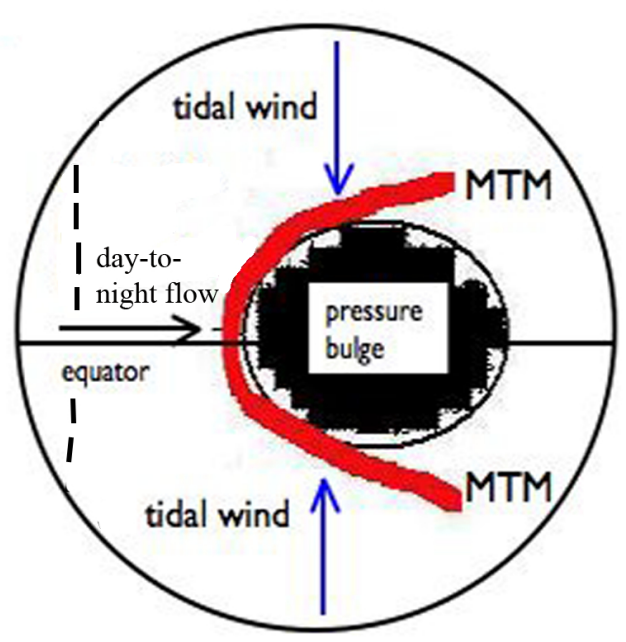

Fig. 12. Comparison of $\mathrm{AE}$ temperature data with a conceptual model showing the MTM formation and the spatial distribution of the midnight pressure bulge. (a) AE-E statistical distribution of the MTM determined for the Southern Hemisphere winter (reproduced from Herrero et al., 1982); (b) conceptual model showing the latitudinal distribution of the MTM formation and spatial distribution plotted against local time. Air moving eastward from the dayside produces converging flow with the thermospheric tidal air flow directed toward the equator from the polar regions in both hemispheres. The red region represents the spatial distribution of the MTM formed by compressional heating in the region of the thermospheric wind convergence. The dashed vertical line refers to the evening twilight terminator.

mated the characteristic time for the pressure to change significantly would be $3 \mathrm{~h}$, but this was for ideal circumstances during solar maximum. Certainly, it is not reasonable to expect that over the vertical extent of the MTM from 200 to $500 \mathrm{~km}$, the downwelling speed would remain constant. 

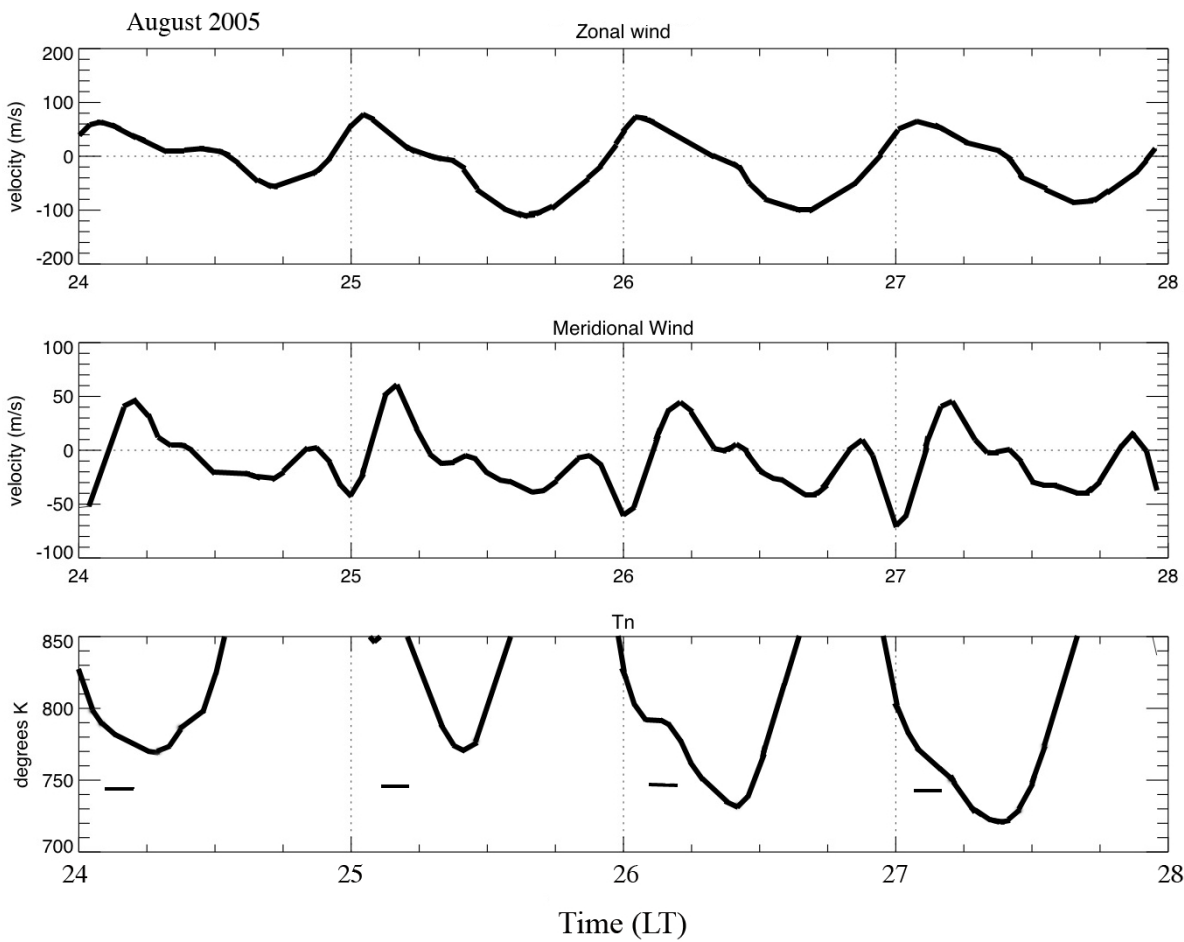

Fig. 13. NCAR TIME-GCM predictions of zonal wind (top panel), meridional wind (middle panel) and temperature (bottom panel) plotted against local time for the location of the Arequipa FPI observatory for the four days from 24 August to 28 August 2005. The horizontal black line located below the temperature plot in each panel marks the expected local time period for the MTM appearance.

Moreover, it is known that the vertical variation of the ion drift speeds changes significantly between $150 \mathrm{~km}$ to $250 \mathrm{~km}$ (Kudeki and Bhattacharyya, 1999). This suggests that the assumption that the vertical variation of the horizontal wind component is constant may not be correct. This last possibility might explain why the vertical wind inferred from the divergence behaves more consistently for the Arecibo observations, which were obtained for solar maximum. In this phase of the solar cycle, the variation of the horizontal wind with altitude is less, and the assumptions used to derive Eq. (7) would be more accurate. It should also be kept in mind that the 630-nm observations we are reporting apply to the region of the thermosphere between 225 and $300 \mathrm{~km}$, over which the measurements of Doppler widths and Doppler shifts are averaged along the instrumental line of sight through the nightglow layer. Quite possibly, the dynamics of the thermosphere observed in this region are not completely representative of the dynamics at higher altitudes; Herrero et al. (1993) noted some differences in the MTM dynamics at heights of 270, 350 , and $440 \mathrm{~km}$, but the overall temporal behavior at each of the three altitudes was similar.

\subsection{Future plans}

Because a major increase in the 630-nm intensity, a factor of 5 , is expected as the solar cycle phase increases toward so- lar maximum, future observations may collect images with shorter exposure times while retaining excellent accuracy. The time remaining in each 30 min cycle could then be used to acquire observations at 4 additional cardinal positions at zenith angles of $45^{\circ}$ within each cycle. The inclusion of additional points will improve the Fourier decomposition procedure so that the horizontal wind maps would portray the wind circulation pattern with more detail.

The increase in 630-nm signal would also provide an opportunity to search for the vertical wind signature associated with the transit of the MTM phenomenon over Arequipa. The localization of the MTM with an amplitude as much as $200 \mathrm{~K}$ over several degrees latitude suggest that the magnitude of the MTM vertical wind might be as detectable if the image exposure times were longer to improve the accuracy. A period of 5 to $10 \mathrm{~ms}^{-1}$ deviation from the longterm trend of the FPI instrumental drift $\left(\sim 100-150 \mathrm{~ms}^{-1}\right.$ per night) would be an indication of such a signature. A search for such vertical winds has been launched at the present time but at solar minimum the $630-\mathrm{nm}$ intensities are weak and success is more problematic.

A second imaging Fabry-Perot observatory will be installed at Huancayo, Peru, with the participation of the research group at the New Jersey Institute of Technology (led by A. Gerrard). Since Huancayo is located on the magnetic 
equator $4^{\circ}$ to the north of the Arequipa site, the combined observations will give a more extended latitudinal range for the horizontal wind and temperature maps, yielding a broader view of the equatorial thermospheric dynamics. Moreover, the two observatories operating in concert as a bistatic observatory (Sipler et al., 1995) will measure directly the neutral wind vector components within one or more common volume regions within the $630-\mathrm{nm}$ nightglow layer. It would then be possible to confirm the validity of the local time and longitude equivalence assumption used for the production of the horizontal wind maps, by comparing the speed and direction of the measured vectors with those inferred from the expansion represented by Eq. (1). It will also be interesting to compare the temporal behavior of the inferred vertical wind for the two sites to assess whether the inferred changes in $w$ are consistent for two observatories which are separated by only $\sim 425 \mathrm{~km}$.

\section{Conclusions}

The Arequipa FPI observations have demonstrated a relationship between the equatorward midnight surge of air that is seen one to two hrs prior to the detection of the MTM peak during its poleward transit over Arequipa. On two occasions when this meridional flow of air is weak, the MTM peak was not identified at all. The passage of the MTM is generally associated with an abatement of the meridional wind component. However, on one occasion near the equinox, the MTM amplitude was very large, $\sim 200-225 \mathrm{~K}$, a reversal in the direction of the meridional flow was seen coincident with the MTM. We conclude that the nighttime meridional flow of air toward the equator is a result of the thermospheric tidal activity that contributes to the formation of the MTM in the region north of the Arequipa site.

Application of the NCAR TIME-GCM model for three nights in late August when the observations indicated the appearance of MTM peaks with amplitudes ranging from 75 to $150 \mathrm{~K}$ showed only a weak indication of the development of the MTM peak. It is not clear whether the inability of the model to simulate the MTM event stems from inadequate spatial resolution in the NCAR model formulation. It may also be the case that the physics involving the coupling of the nighttime F-region to the thermosphere is not modeled correctly. Clearly, the MTM phenomenon is spatially confined within a distance of 300 to $500 \mathrm{~km}$ which is less than the model grid point resolution of $500 \mathrm{~km}$. In addition to this improvement of increased spatial resolution, the model may also need to include specifically a hydrodynamical mechanism to describe more accurately the physics of the merging of the nighttime eastward zonal wind flow with the tidal wind equatorward flow. Finally, we note that the NCAR model has the compressional heating taking place coincident in space with the region of the pressure bulge. Consequently, the scale of the model MTM as illustrated in Colerico et al. (2006) is much larger than the observed scale. As Fig. 12b illustrates, the MTM heating takes place along the perimeter of the pressure bulge rather than within the bulge. Thus, the MTM is localized within the distance of a few hundred kilometers. Thus, one can consider that the problem with the TIME-GCM reproducing the MTM phenomenon has more to do with the calculations of its latitudinal spatial distribution rather than the determination of the energy required for the compressional heating. The lack of any terdiurnal tidal forcing which is more localized in scale might account for this shortcoming of the model.

Acknowledgements. The authors thank M. A. Biondi for his contributions regarding the experimental modifications reported here. J. Makela (Univ. of Illinois) provided helpful comments regarding the manuscript. Support for this research was provided by the National Science Foundation grants to Clemson University and Dartmouth College. The authors acknowledge with thanks the support provided by the National Aeronautics Space Administration (NASA) Satellite Laser Ranging (SLR) Program and to the Universidad Nacional de San Agustin (UNSA) located in Arequipa, Peru, for their hospitality in providing the use of the San Francisco Observatory station in Characato, Peru, for the acquisition of these measurements.

Topical Editor U.-P. Hoppe thanks two anonymous referees for their help in evaluating this paper.

\section{References}

Arduini, C., Laneve, G., and Herrero, F. A.: Local time and altitude variation of equatorial thermosphere midnight density maximum (MDM): San Marco drag balance measurements, Geophys. Res. Lett., 24, 377-380, 1997.

Aruliah, A. I. and Griffin, E. M.: Evidence of meso-scale structure in the high-latitude thermosphere, Ann. Geophys., 19, 3746, 2001, http://www.ann-geophys.net/19/37/2001/.

Aruliah, A. I., Griffin, E. M., Aylward, A. D., Ford, E. A. K., Kosch, M. J., Davis, C. J., Howells, V. S. C., Pryse, S. E., Middleton, H. R., and Jussila, J.: First direct evidence of meso-scale variability on ion-neutral dynamics using co-located FPIs and EISCAT radar in Northern Scandinavia, Ann. Geophys., 23, 147182, 2005, http://www.ann-geophys.net/23/147/2005/.

Bamgboye, D. K. and McClure, J. P.: Seasonal variation in the occurrence time of the equatorial midnight temperature bulge, Geophys. Res. Lett., 9, 457-460, 1982.

Behnke, R. A. and Harper, R. M.: Vector measurements of F region ion transport at Arecibo, J. Geophys. Res., 78, 8222-8234, 1973.

Bevington, P. R. and Robinson, D. K.: Data Reduction and Error Analysis for the Physical Sciences, McGraw-Hill, New York, 1992.

Biondi, M. A. and Meriwether, J. W.: Measured response of the equatorial ther-mospheric temperature to geomagnetic and solar flux changes, Geophys. Res. Lett., 12, 267-270, 1985.

Biondi, M. A., Sipler, D. P., and Weinschenker, M.: Multiple aperture exit plate for field-widening a Fabry-Perot interferometer, Appl. Optics, 24, 232-238, 1985.

Biondi, M. A., Sazykin, S. Y., Fejer, B. G., Meriwether, J. W., and Fesen, C. G.: Equatorial and low-latitude thermospheric winds: 
Measured quiet time variations with season and solar flux from 1980 to 1990, J. Geophys. Res., 104, 17 091-17 106, 1999.

Burnside, R. G., Herrero, F. A., Meriwether, J. W., and Walker, J. C. G.: Optical observations of thermospheric dynamics at Arecibo, J. Geophys. Res., 86, 5532-5540, 1991.

Coakley, M. M., Roesler, F. L., Reynolds, R. J., and Nossal, S.: Fabry-Perot CCD annular-summing spectroscopy: study and implementation for aeronomy applications, Appl. Optics, 35, 64796493, 1996.

Colerico, M., Mendillo, M., Nottingham, D., Baumgardner, J., Meriwether, J., Mirick, J., Reinish, B., Scali, J., and Fesen, C.: Coordinated measurements of F-region dynamics related to the thermospheric midnight temperature maximum, J. Geophys. Res., 101, 26783-26 794, 1996.

Colerico, M. and Mendillo, M.: The current state of investigations regarding the thermospheric midnight temperature maximum (MTM), J. Atmos. Solar Terr. Phys., 64, 1361-1369, 2002.

Colerico, M., Mendillo, M., Fesen, C. G., and Meriwether, J. W.: Comparative investigations into the equatorial electrodynamics and low to mid latitude coupling of the thermosphere-ionosphere system in the American sector, Ann. Geophys., 24, 503-513, 2006, http://www.ann-geophys.net/24/503/2006/.

Conde, M.: Deriving wavelength spectra from fringe images from a fixed-gap single-etalon Fabry-Perot spectrometer, Appl. Optics, 41, 2672-2678, 2002.

Crary, D. J. and Forbes, J. M.: The dynamic ionosphere over Arecibo: A theoretical investigation, J. Geophys. Res., 91, 249258, 1986

Faivre, M., Meriwether, J. W., Fesen, C. G., and Biondi, M. A.: Climatology of the midnight temperature maximum phenomenon at Arequipa, Peru, J. Geophys. Res., 111, A06302, doi:10.1029/2005JA011321, 2006.

Fesen, C. G.: Simulations of the low-latitude midnight temperature maximum, J. Geophys. Res., 101, 26 863-26 874, 1996.

Fesen, C. G.: Theoretical effects of tides and geomagnetic activity in the low latitude ionosphere, J. Atmos. Terr. Phys., 59, 15211531, 1997.

Harper, R. M.: Nighttime meridional neutral winds near $350 \mathrm{~km}$ at low to mid-latitudes, J. Atmos. Terr. Phys., 35, 2023-2034, 1973.

Hays, P. B. and Roble, R. G.: A technique for recovering Doppler line profiles from Fabry-Perot interferometer fringes of very low intensity, Appl. Optics, 10, 193-200, 1971.

Hernandez, G.: Fabry-Perot interferometers, Cambridge and New York, Cambridge University Press (Cambridge Studies in Modern Optics, No. 3), 1986.

Herrero, F. A. and Spencer, N. W.: On the horizontal distribution of the equatorial thermospheric midnight temperature maximum and its seasonal variation, Geophys. Res. Lett., 9, 1179-1182, 1982.

Herrero, F. A., Mayr, H. G., and Spencer, N. W.: Latitudinal (Seasonal) variations in the thermospheric midnight temperature maximum: a tidal analysis, J. Geophys. Res., 88, 7225-7235, 1983.

Herrero, F. A., Spencer, N. W., and Mayr, H. G.: Thermosphere and F-region plasma dynamics in the equatorial region, Adv. Space Res., 13, 201-220, 1993.

Killeen, T. L. and Hays, P. B.: Doppler line profile analysis for a multi-channel Fabry-Perot interferometer, Appl. Optics, 23, 612-620, 1984
Kudeki, E. and Bhattacharyya, S.: Postsunset vortex in equatorial F-region plasma drifts and implications for bottomside spread-F, J. Geophys. Res., 104, 28 163-28 170, 1999.

Martinis, C., Baumgardner, J., Smith, S. M., Colerico, M., and Mendillo, M.: Imaging science at El Leoncito, Argentina, Ann. Geophys., 24, 1375-1385, 2006, http://www.ann-geophys.net/24/1375/2006/.

Mayr, H. G., Harris, I., Spencer, N. W., Hedin, A. E., Wharton, L. E., Porter, H. S., Walker, J. C. G., and Carlson, H. C.: Atmospheric tides and the midnight temperature anomaly in the thermosphere, Geophys. Res. Lett., 6, 447-450, 1979.

Meriwether, J. W., Moody, J. W., Biondi, M. A., and Roble, R. G.: Optical interferometric measurements of nighttime equatorial thermospheric winds at Arequipa, Peru, J. Geophys. Res., 91, 5557-5566, 1986.

Meriwether Jr., J. W. and Shih, P.: On the nighttime signatures of thermospheric winds observed at Sondrestrom, Greenland, as correlated with interplanetary magnetic field parameters, Ann. Geophys., 5, 329-336, 1987,

http://www.ann-geophys.net/5/329/1987/.

Meriwether, J. W., Biondi, M. A., Herrero, F. A., Fesen, C. G., and Hallenback, D. C.: Optical interferometric studies of the nighttime equatorial thermosphere: Enhanced temperature and zonal wind gradients, J. Geophys. Res., 102, 20 041-20 058, 1997.

Mierkiewicz, E. J., Roesler, F. L., Nossal, S. M., and Reynolds, R. J.: Geocoronal hydrogen studies using Fabry-Perot interferometers, part 1: Instrumentation, observations, and analysis, J. Atmos. Solar-Terr. Phys., 68, 1520-1519, 2006.

Nelson, G. J. and Cogger, L. L.: Dynamical behavior of the nighttime ionosphere at Arecibo, J. Atmos. Terr. Phys., 33, 17111726, 1971.

Nossal, S. M., Mierkiewicz, E. J., Roesler, F. L., Reynolds, R. J., and Haffner, L. M.: Geocoronal hydrogen studies using FabryPerot interferometers, part 2: Long-term observations, J. Atmos. Solar-Terr. Phys., 68, 1553-1575, 2006.

Picone, M., Hedin, A. E., Drob, D. P., and Aikin, A. C.: NRLMSISE-00 empirical model of the atmosphere: Statistical comparisons and scientific issues, J. Geophys. Res., 107(A12), 1468, doi:10.1029/2002JA009430, 1971.

Roble, R. G.: The NCAR Thermosphere-Ionosphere-MesosphereElectrodynamics General Circulation Model (TIME-GCM), Ionosphere Models, STEP Handbook on Ionospheric Models, edited by: Schunk, R. W., Utah State University, 1996.

Roble, R. G. and Ridley, E. C.: A thermosphere-ionospheremesosphere-electrodynamics general circulation model (TIMEGCM): Equinox solar cycle minimum simulations $(30-500 \mathrm{~km})$, Geophys. Res. Lett., 21, 417-420, 1994.

Roble, R. G., Ridley, E. C., Richmond, A. D., and Dickinson, R. E.: A coupled thermosphere/ionosphere general circulation model, Geophys. Res. Lett., 15, 1325-1328, 1988.

Sipler, D. P., Biondi, M. A., and Zipf, M. E.: Vertical winds in the midlatitude thermosphere from Fabry-Perot Interferometer measurements, J. Atmos. Terr. Phys., 57, 621-629, 1995.

Spencer, N. W., Carignan, G. R., Mayr, H. G., Neimann, B. H., Theis, R. F., and Wharton, L. E.: The midnight temperature maximum in the Earth's equatorial thermosphere, Geophys. Res. Lett., 6, 444-446, 1979. 